\title{
Intelligent Backpropagated Neural Networks for Numerical Computations for MHD Squeezing Fluid Suspended by Nanoparticles between Two Parallel Plates
}

\section{Muhammad Shoaib}

COMSATS University Islamabad

Muhammad Asif Zahoor Raja

National Yunlin University of Science and Technology

Imrana Farhat

COMSATS University Islamabad

Zahir Shah ( zahir1987@yahoo.com )

King Mongkut's University of Technology Thonburi

Poom Kumam

King Mongkut's University of Technology Thonburi

\section{Research Article}

Keywords: MHD nanofluid, artificial intelligence based numerical computation, Levenberg Marquardt algorithm, backpropagated neural network, squeezing 2D flow

Posted Date: January 13th, 2021

DOl: https://doi.org/10.21203/rs.3.rs-139804/v1

License: (c) (i) This work is licensed under a Creative Commons Attribution 4.0 International License.

Read Full License 


\title{
Intelligent Backpropagated Neural Networks for numerical computations for MHD squeezing fluid suspended by nanoparticles between two parallel plates
}

Muhammad Shoaib ${ }^{1}$, Muhammad Asif Zahoor Raja ${ }^{2,3}$, Imrana Farhat ${ }^{1}$, , Zahir Shah ${ }^{4,5}$, Poom Kumam $^{5,6}$

${ }^{1}$ Department of Mathematics, COMSATS University Islamabad, Attock Campus, Pakistan

${ }^{2}$ Future Technology Research Center, National Yunlin University of Science and Technology, 123 University Road, Section .3, Douliou, Yunlin 64002, Taiwan, R.O.C.

${ }^{3}$ Department of Electrical and Computer Engineering, COMSATS University Islamabad, Attock Campus, Pakistan

${ }^{4}$ Department of Mathematics, University of Lakki Marwat, Lakki Marwat 28420, Khyber Pakhtunkhwa Pakistan

${ }^{5}$ Center of Excellence in Theoretical and Computational Science (TaCS-CoE), Faculty of Science, King Mongkut's University of Technology Thonburi (KMUTT), 126 Pracha Uthit Rd., Bang Mod, Thung Khru, Bangkok 10140, Thailand

${ }^{6}$ Fixed Point Research Laboratory, Fixed Point Theory and Applications Research Group, Center of Excellence in Theoretical and Computational Science (TaCS-CoE), Faculty of Science, King Mongkut's University of Technology Thonburi (KMUTT), 126 Pracha Uthit Rd., Bang Mod, Thung Khru, Bangkok 10140, Thailand

Corresponding authors: Poom Kumam(poom.kum@kmutt.ac.th) and Zahir Shah (zahir@ulm.edu.pk)

\begin{abstract}
:
In the present research, artificial intelligence based backpropagated neural networks with Levenberg-Marquardt algorithm (BNN-LMA) are utilized to interpret the numerical computation for squeezing 2D magneto-hydrodynamic (MHD) nanofluid flow between two parallel plates. The non-linear system of ODEs represents the magneto-hydrodynamic, squeezing nanofluidic flow model (MHD-SNFM). A reference dataset for BNN-LMA is formulated by utilizing Adam numerical solver for different scenarios of MHD-SNFM by variation of squeezing number, Hartmann Number and heat source parameter. The validation, testing and training processes of BNN-LMA are exploited to analyze the approximate solution of MHD-SNFM for different scenarios and correctness of proposed BNN-LMA is verified by comparison of reference outcomes. The performance of BNN-LMA to solve the MHD-SNFM is validated through regression analysis, histogram studies and mean square error (MSE).
\end{abstract}

Keywords- MHD nanofluid, artificial intelligence based numerical computation, Levenberg Marquardt algorithm, backpropagated neural network, squeezing 2D flow. 


\section{Introduction:}

The squeezing flow, first explored by Josef Stefen, is the form of flow in which flow is squeezed, deformed or pressed out between two parallel plates or disks. Moreover, it demonstrates the movement of fluid particles, its contact with the surface of plates and effects of other parameters such as temperature, viscosity and heat source parameter etc. In this research we are dealing with nanofluid squeezed between two parallel particles, nanofluid comprises of nanoparticles mixed base fluids. These nanoparticles are metal oxides, metals or carbon nanotubes.

In the recent years, most of the literature has been done on study of nano-particles and nanofluids. Nanofluids, first named by Choi [1], are basically nanoparticles suspended in base fluids. T Hayat et. al [2] have studied the behavior of parameters on velocity, temperature and concentration profiles, also computed and analyzed the heat and mass transfer. The fluid flow and heat transfer characteristics are analyzed by Mehmood et al. [3]. Siddique et al. [4] analyses the MHD squeezing flow between two parallel surfaces. Shoaib et al. [5] investigated the heat and mass transfer in 3-D MHD radiative flow of of hybrid nanofluid. If one nanoparticle is mixed with base fluid, it is mono nanofluid while if two or more nanoparticles are added then it is hybrid nanofluid. Ali Imran et al. [6] theoretically investigate the heat transfer of nanofluid flow in ciliated channel.

Azimi et al. [7] have discussed MHD squeezing flow of nanofluid between parallel plates and compared the analytical and numerical results. The heat transfer in unsteady nanofluid flow between two moveable parallel plates is investigated by Ganji DD et al. [8], and analyzed the effects of various parameters. The chemical reactions, velocity slip, thermal radiation and Brownian motion in 3-D flow of Casson nanofluid has been studied by M Umar et al. [9]. Babazadeh et al. [10] have studied the effects of thermophoresis, magnetic forces on nanoparticles squeezed between two plates. Noor et al. [11-12] have analyzed the unsteady MHD squeezing flow of Jeffery fluid in a porous medium and effects of viscous dissipation and chemical reaction on MHD squeezing flow of Casson nanofluid between parallel plates in a porous medium. Many of other researchers [13-21] have contributed their work on squeezing flow of nanofluids including hybrid nanofluids and Casson nanofluid.

In most of the engineering problems, the system of PDEs representing mathematical relations of the problem are transformed into ODEs. In many cases, the solution to scientific problems does not admitted analytically, this leads to the equation to be solved by using special techniques. One of the methods are reconstruction of variational iteration method [22], homotopy perturbation method [23] and other [24-25]. Later many other researchers worked on other techniques, including Jadoonet al. [26] and Oyang et al. [27] and many other [28-34] to solve the many problems.

The optimization procedures including evolutionary computation techniques are utilized in stochastic numerical computing solvers connected with neural networks for determining the results/ solutions of linear or non-linear differential equations representing different models of the problem. Recently, the implementation of these techniques include thermodynamics [35], 
atomic physics [36-37], magneto-hydrodynamics [38-39], fluid dynamics [40-41] and nanotechnology [42-43].

Although above cited literature on nanofluid flows containing nanoparticles for different fluidic systems, mostly on squeezing flows by using various traditional numerical and analytical methods; but stochastic numerical techniques are required to exploit for squeezing flow problems due to their worth, effectiveness and robustness. The stochastic numerical methods are already implemented for various research problems by the research workers [44-49]. Some most recent artificial intelligence based techniques are Emden-Fowler Model [50], nonlinear unipolar electro hydrodynamic pump flow model [51], non-linear corneal shape model [52] and COVID-19 Models [53-54]. These soft computing infrastructures are inspiring factors for the authors source of motivation to exploit an accurate and reliable alternate framework based on soft computing infrastructure for the solution of heat generation in mixed convected Williamson fluid stretching flow problem by conducting a parametric study to examine the effects or various physical quantities on the velocity, concentration and temperature profiles. Mathematica and MATLAB software are utilized for numerical treatment.

The experimental insights of computing simulation are highlighted as follows:

- Intelligent computation is presented by Levenberg-Marquardt algorithm based backpropagated neural networks to study the MHD squeezing nanofluid flow between two parallel plates.

- The designed BNN-LMA coupled with PDEs for the MHD-SNFM, which are then converted into system of ODEs.

- Adams numerical techniques is used to interpret the dataset for designed BNN-LMA for the variants of MHD-SNFM on the basis of squeezing number, Hartmann Number and heat source parameter.

- The training, validation and testing processes of BNN-LMA are utilized by modelling MHD squeezing nanofluid flow model for different scenarios.

The performance of designed BNN-LMA validated through convergence plots of MSE, error histograms, regression and fitness function.

\section{Problem Formulation of MHD-SNFM:}

In this research, numerical computation for the analysis of 2D unsteady, incompressible, squeezing nanofluid flow between two parallel plates having distance $z= \pm l \sqrt{1-a t}=$ $\pm h(t)$ has been interpreted, where $l$ is the distance between the plates at $t=0$, and $a$ is squeezed parameter, when $a>0$ the plates are squeezed but when $a<0$ and $t=\frac{1}{a}$ the plates are separated. The magnetic field's variation with time $t$ is defined as $B=$ $B_{0}(1-a t)$, heat generation is neglected due to viscous dissipation effect in the flow. There is nanofluid flow flowing between the plates have following assumption: radiative heat transfer is neglected, no chemical reaction and no-slip condition occurs. The nanoparticles and base flow that is incompressible are in thermal equilibrium. Figure 1 shows the geometry of the given problem. 

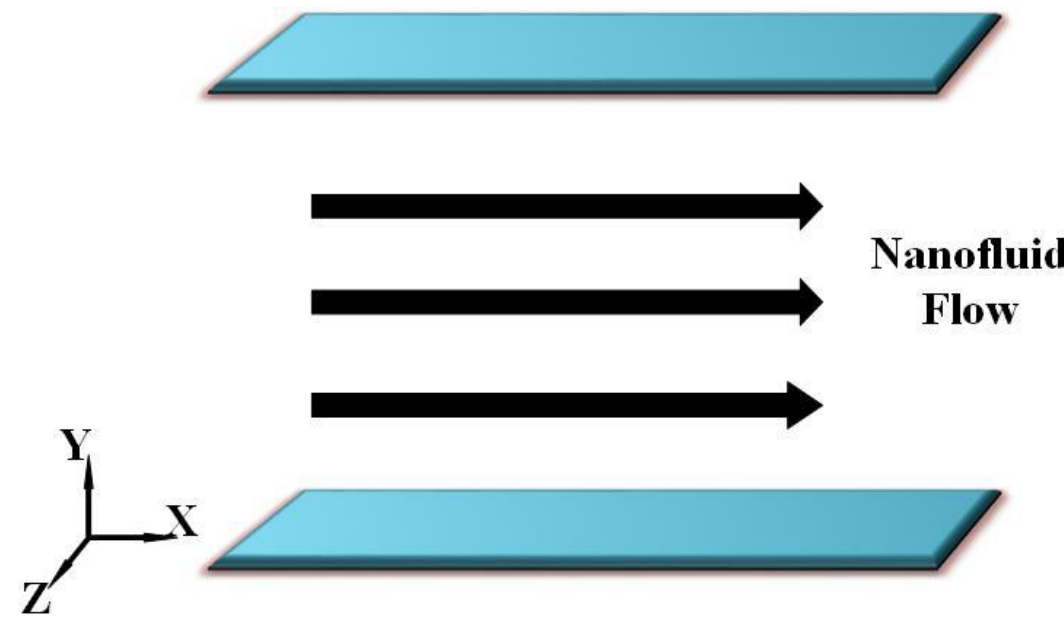

Figure 1: MHD Squeezing fluid flow between two parallel plates

The governing equations of conservation of momentum and energy are given as [55]:

$$
\begin{aligned}
& \frac{\partial u}{\partial x}+\frac{\partial v}{\partial y}=0 \\
& \rho_{h n f}\left(\frac{\partial u}{\partial t}+u \frac{\partial u}{\partial x}+v \frac{\partial u}{\partial y}\right)=-\frac{\partial p}{\partial x}+\mu_{h n f}\left(\frac{\partial^{2} u}{\partial x^{2}}+\frac{\partial^{2} u}{\partial y^{2}}\right)-\sigma_{h n f} B^{2} u \\
& \rho_{h n f}\left(\frac{\partial v}{\partial t}+u \frac{\partial v}{\partial x}+v \frac{\partial v}{\partial y}\right)=-\frac{\partial p}{\partial y}+\mu_{h n f}\left(\frac{\partial^{2} v}{\partial x^{2}}+\frac{\partial^{2} v}{\partial y^{2}}\right) \\
& \frac{\partial T}{\partial t}+u \frac{\partial T}{\partial x}+v \frac{\partial T}{\partial y}=\frac{k_{h n f}}{\left(\rho C_{p}\right)_{h n f}}\left(\frac{\partial^{2} T}{\partial x^{2}}+\frac{\partial^{2} T}{\partial y^{2}}\right)+\frac{Q}{\left(\rho C_{p}\right)_{h n f}} T
\end{aligned}
$$

Where $\mathrm{u}$ and $\mathrm{v}$ are velocity components, $\mathrm{T}$ is temperature, $p$ pressure, $Q$ is function of heat source, $\rho_{h n f}$ effective density, $\left(\rho C_{p}\right)_{h n f}$ effective heat capacity and $\sigma_{h n f}$ is electrical conductivity of nanofluid. The boundary conditions are:

$$
\begin{aligned}
& v=v_{w}=\frac{d h}{d t}, \quad T=T_{H}, \quad y=h(t), \\
& v=\frac{\partial u}{\partial y}=\frac{\partial t}{\partial y}=0 y=0 .
\end{aligned}
$$

Now by using some parameters given below:

$$
\eta=\frac{y}{l \sqrt{1-a t}}, \quad u=\frac{a x}{l(1-a t)} f^{\prime}(\eta), \quad v=\frac{a l}{l \sqrt{1-a t}}, \quad \theta=\frac{T}{T_{H}},
$$

The system of PDEs are transformed into system of ODEs as follows: 


$$
\begin{aligned}
& f^{i v}-S\left(\frac{A_{1}}{A_{4}}\right)\left(\eta f^{\prime \prime \prime}+3 f^{\prime \prime}+f^{\prime} f^{\prime \prime}-f f^{\prime \prime \prime}\right)-H a^{2}\left(\frac{A_{5}}{A_{4}}\right) f^{\prime \prime}=0, \\
& \theta^{\prime \prime}+\operatorname{Pr} S\left(\frac{A_{2}}{A_{3}}\right)\left(f \theta^{\prime}-\eta \theta^{\prime}\right)+\frac{H s}{A_{3}} \theta=0,
\end{aligned}
$$

And the boundary conditions are:

$$
\begin{gathered}
f(0)=0, \quad f^{\prime}(0)=0, \\
f(1)=1, \quad f^{\prime}(1)=0, \\
\theta^{\prime}(0)=0, \quad \theta(1)=1 .
\end{gathered}
$$

Where $\mathrm{S}$ is squeezing number, $\mathrm{Ha}$ is Hartmann Number, $\mathrm{Pr}$ is Prandtl number and $\mathrm{Hs}$ is heat source parameter and $A_{1}, A_{2}, A_{3}, A_{4}$ and $A_{5}$ are dimensional constants.

\section{Solution Methodology:}

The nftool, an effective algorithm in artificial based neural networks (NNs) toolbox in MATLAB software package is utilized to execute the designed backpropagated neural network with Levenberg Marquardt Algorithm (BNN-LMA). The solution methodology consists of essential description for dataset and implementation procedure for implementation of designed BNNLMA. The designed neural network for BNN-LMA is shown in Figure 2 and flow chart of methodology presented in Figure 3.

Table 1. Depiction of scenarios for MHD-SNFM

\begin{tabular}{|c|c|c|c|c|}
\hline \multirow{3}{*}{ Scenarios } & \multirow{2}{*}{ Cases } & \multicolumn{3}{|c|}{ Physical Quantities } \\
\cline { 2 - 5 } & & $\mathrm{S}$ & Ha & Hs \\
\hline \multirow{4}{*}{ Scenario 1 } & 1 & 1 & 0 & -1 \\
\cline { 2 - 5 } & 2 & 3 & 0 & -1 \\
\cline { 2 - 5 } & 3 & 5 & 0 & -1 \\
\cline { 2 - 5 } & 4 & 7 & 0 & -1 \\
\hline \multirow{4}{*}{ Scenario 2 } & 1 & 1 & 0 & -1 \\
\cline { 2 - 5 } & 2 & 1 & 2 & -1 \\
\cline { 2 - 5 } & 3 & 1 & 3 & -1 \\
\hline \multirow{4}{*}{ Scenario 3 } & 4 & 1 & 4 & -1 \\
\cline { 2 - 5 } & 2 & 1 & 0 & -1 \\
\cline { 2 - 5 } & 3 & 1 & 0 & -3 \\
\cline { 2 - 5 } & 4 & 1 & 0 & -7 \\
\hline
\end{tabular}


Mathematical relation of MHD-SNFM model (7-9) is presented for case 1 of scenario 1.

$$
\begin{aligned}
& f^{i v}-2\left(\eta f^{\prime \prime \prime}+3 f^{\prime \prime}+f^{\prime} f^{\prime \prime}-f f^{\prime \prime \prime}\right)=0, \\
& \theta^{\prime \prime}+0.625\left(f \theta^{\prime}-\eta \theta^{\prime}\right)-0.312 \theta=0,
\end{aligned}
$$

And the boundary conditions are:

$$
\begin{array}{ll}
f(0)=0, & f^{\prime}(0)=0, \\
f(1)=1, & f^{\prime}(1)=0, \\
\theta^{\prime}(0)=0, & \theta(1)=1 .
\end{array}
$$

Similarly, we can obtain mathematical relations for different cases of all the scenarios in designed MHD-SNFM.

The reference dataset of designed BNN-LMA is created for inputs between 0 and 1 with time interval of 0.01 by utilizing Adam numerical solver through "NDSolve" in Mathematica software package by variation of squeezing number, Hartmann Number and heat source parameter in MHD-SNFM as listed below in the Table 1.

\section{Interpretation of Results:}

The possible outcomes of numerical computation for the designed neural networks backpropagated with Levenberg Marquardt algorithm is exploited for the proposed MHD-SNFM as presented in equations (7-9). The three scenarios of MHD-SNFM by variation of squeezing number S, Hartmann Number Ha and heat source parameter Hs are formulated for four different cases for both velocity and temperature parameters of MHD squeezing nanofluidic flow model as mentioned in Table 1.

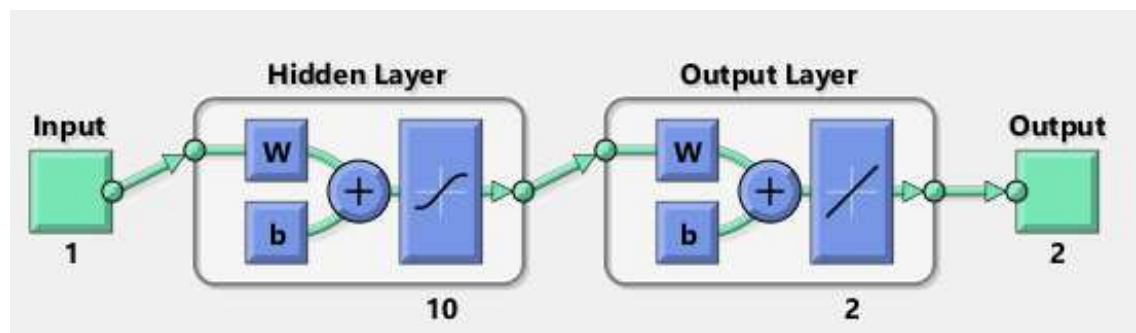

Figure 2 Neural Network for designed BNN-LMA of MHD-SNFM

The reference dataset for $f(\eta)$ and $\theta(\eta)$ of designed BNN-LMA are determined by utilizing Adams numerical method for $\eta$ between 0 and 1, with step size of 0.01 for all the four cases of three different scenarios of BNN-LMA of MHD-SNFM. The obtained dataset in terms of $f(\eta)$ and $\theta(\eta)$ is then utilized as reference outcome in this presented research.

The designed BNN-LMA is exploited to determine the solution of squeezing, nanofluidic flow model in between two parallel plates by utilizing nftool in MATLAB software package. The reference dataset for velocity and temperature profiles $(f$ and $\theta)$ is created for 101 input points in 
which $80 \%$ are utilized for training, $10 \%$ each for validation and testing respectively for BNNLMA using the neural network as presented in Figure 3.

The solutions of BNN-LMA for second case of each scenario in terms of performance and state transition is represented in Figures 4-5 and function fitness with error histogram plots are demonstrated in Figure 6 for second case of each scenario while regression plots are depicted in Figure 7. Moreover, the convergence in terms of MSE for performance of training, testing and validation, performance, epochs, backpropagated operator i-e., $\mathrm{Mu}$ and time taken are presented in Tables 2-4.

In the subfigures 4(a), 4(b), 4(c), the convergence of MSE for training, testing and validation curves are depicted for second case of all the scenarios of MHD-SNFM. The most excellent execution is accomplished at 134, 127 and 166 epochs with MSE around $10^{-11}, 10^{-11}$ and $10^{-09}$ respectively. The gradient and $\mathrm{Mu}$ parameter of Levenberg Marquardt backpropagation are [9.84E-08, 9.88E-08 and 9.91E-08] and [1.00E-10, 1.00E-10 and 1.00E-09] as demonstrated in subfigures 5(a), 5(b) and 5(c). The correctness and convergent efficiency of BNN-LMA for each case of MHD-SNFM has been proved by outcomes.

The efficiency of results of BNN-LMA is observed with matching outcomes of Adam numerical solver for all the three scenarios of MHD-SNFM as shown in Figure 6 which is further endorsed by error plots. The investigation through regression analysis is carried out by co-relation studies. Figure 7 shows the results of regression outcomes of respective three variants of MHD-SNFM. One may see that the value of correlation $\mathrm{R}$ close to unity indicates perfect modeling, in terms of training, testing and validation certified the correctness of BNN-LMA for the designed MHDSNFM.

Additionally, the corresponding numerical values listed in Tables 2-4 for all the three scenarios of both velocity and temperature profiles of designed MHD-SNFM demonstrated the performance on MSE around E-11 to E-12, E-11 to E-12 and E-09 to E-11. The consistent and accurate performance of BNN-LMA is authenticated by all the numerical illustrations in Tables 2-4 for solving each variant of MHD-SNFM.

Consequently, the solutions of BNN-LMA are executed for the velocity profile $f(\eta)$ for all the three scenarios of MHD-SNFM, demonstrated in subfigures 8(a), 8(c) and 8(e), while the solutions for $\theta(\eta)$ are depicted in 9(a), 9(c) and 9(e). One can witness that Absolute Error achieved for $f(\eta)$ are about $10^{-4}$ to $10^{-09}, 10^{-4}$ to $10^{-08}$ and $10^{-3}$ to $10^{-08}$ of scenario 1 , scenario 2 and scenario 3 respectively, as shown in 8(b), 8(d) and 8(f). And the Absolute Error achieved for $\theta(\eta)$ are about $10^{-5}$ to $10^{-08}, 10^{-5}$ to $10^{-08}$ and $10^{-4}$ to $10^{-09}$ of scenario 1 , scenario 2 and scenario 3 respectively, as shown in 9(b), 9(d) and 9(f). Moreover, one can observe from subfigures 8(a), 8(c) and 8(e), the velocity profile increases with the increase in squeeze number and decreases with increase in Hartmann number, while subfigures 9(a), 9(c) and $9(\mathrm{e})$, the temperature profile decreases with the increase in squeeze number and Hartmann number. 


\section{The Problem Development}

\section{MHD squeezing fluid suspended by nanoparticles between two parallel plates}

System of PDEs representing MHD-SNFM is converted into system of ODEs

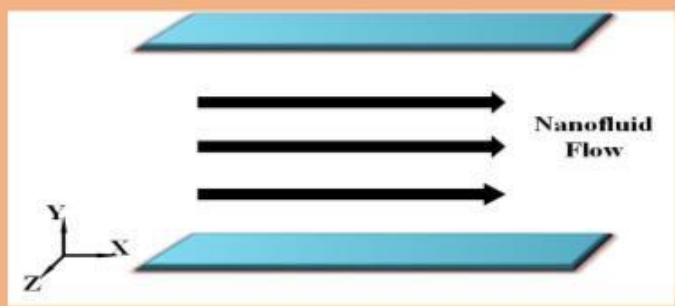

Geometry of the Problem

\section{Design Scheme for BNN-LMA of proposed MHD-SNFM}

\section{Adam Numerical Outcomes:}

Adam numerical solver is utilized to generate the reference dataset by variation of squeezing number.

Hartmann number and heat source parameter of MHD-SNFM
Backpropagated neural networks:

Artificial intelligence based numerical computing technique is devel oped by using neural networks

backpropagated with Levenberg-Marquardt algorithm.

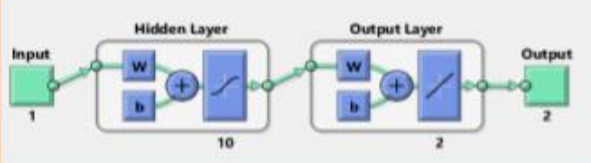

Neural Netw ork for designed BNN-LMA of MHD-SNFM

\section{Analysis of Reference Dataset}

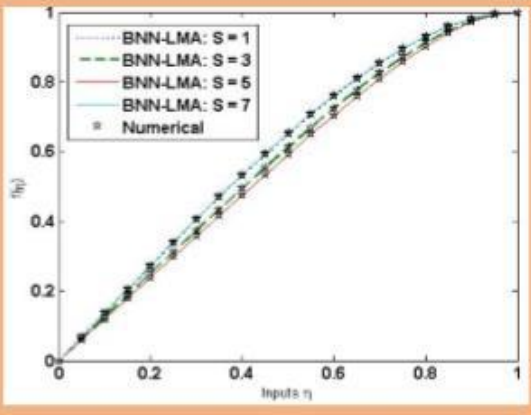

Outcomes with comparison

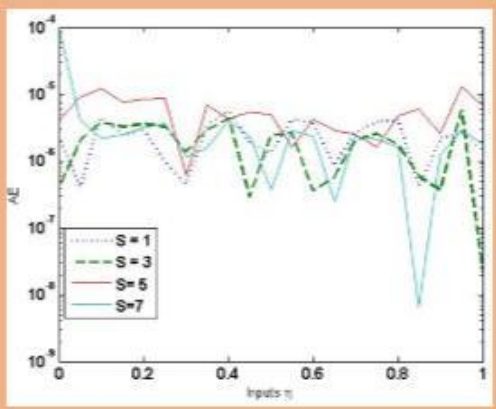

Error Analysis

Figure 3 Flow Chart of MHD Squeezing fluid flow model 


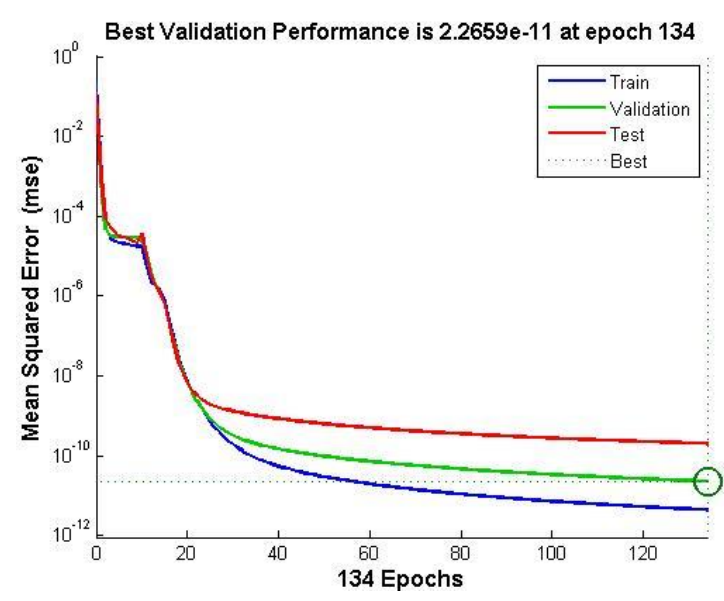

a) Results of MSE for Case 2 of Scenario 1

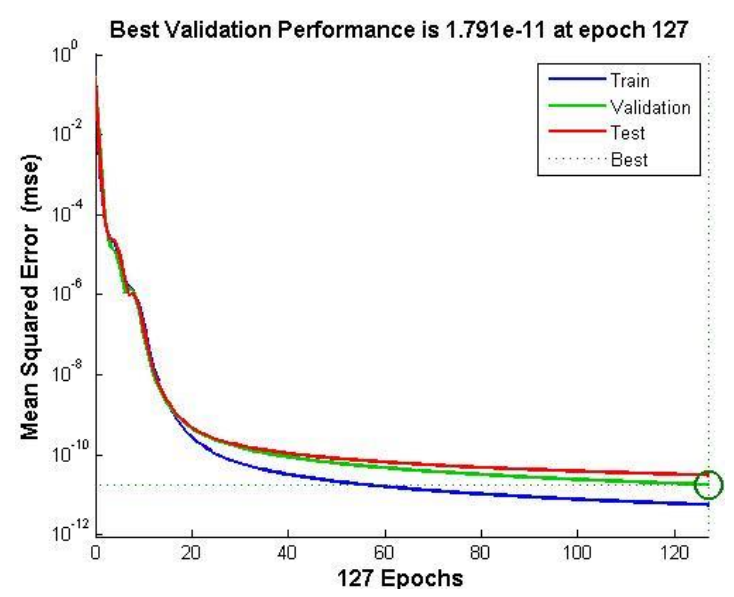

b) Results of MSE for Case 2 of Scenario 2

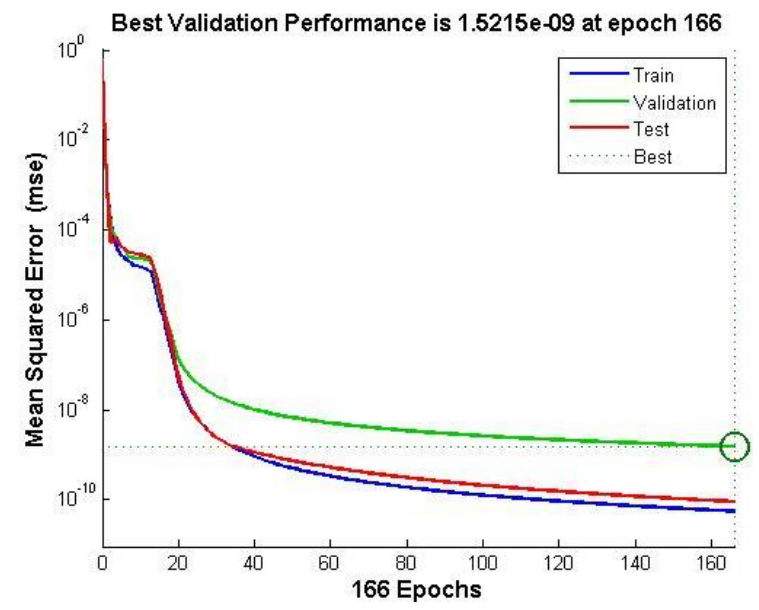

c) Results of MSE for Case 2 of Scenario 3

Figure 4: Performance on MSE of designed BNN-LMA of case 2 of all the scenarios of MHD-SNFM

Table 2: Outcomes of BNN-LMA for Scenario 1 of MHD-SNFM

\begin{tabular}{|c|c|c|c|c|c|c|c|c|c|}
\hline \multirow[t]{2}{*}{ Case } & \multirow{2}{*}{$\begin{array}{l}\text { Hidden } \\
\text { neurons }\end{array}$} & \multicolumn{3}{|c|}{ MSE } & \multirow[t]{2}{*}{ Performance } & \multirow[t]{2}{*}{ Grad } & \multirow[t]{2}{*}{$\mathrm{Mu}$} & \multirow[t]{2}{*}{ Epochs } & \multirow[t]{2}{*}{ Time } \\
\hline & & Training & Validation & Testing & & & & & \\
\hline 1 & 10 & $6.69 \mathrm{E}-12$ & $1.60 \mathrm{E}-11$ & $2.22 \mathrm{E}-11$ & $6.70 \mathrm{E}-12$ & $\begin{array}{c}9.95 \mathrm{E}- \\
08 \\
\end{array}$ & $\begin{array}{l}1.00 \mathrm{E} \\
-10 \\
\end{array}$ & 117 & $3 \mathrm{~s}$ \\
\hline 2 & 10 & $4.36 \mathrm{E}-11$ & $2.27 \mathrm{E}-11$ & $2.00 \mathrm{E}-10$ & $4.36 \mathrm{E}-12$ & $\begin{array}{c}9.84 \mathrm{E}- \\
08 \\
\end{array}$ & $\begin{array}{l}1.00 \mathrm{E} \\
-10 \\
\end{array}$ & 134 & $1 \mathrm{~s}$ \\
\hline 3 & 10 & $3.44 \mathrm{E}-11$ & $1.27 \mathrm{E}-10$ & $5.84 \mathrm{E}-11$ & $3.44 \mathrm{E}-11$ & $\begin{array}{c}9.96 \mathrm{E}- \\
08 \\
\end{array}$ & $\begin{array}{c}1.00 \mathrm{E} \\
-09 \\
\end{array}$ & 201 & $2 \mathrm{~s}$ \\
\hline 4 & 10 & $4.65 \mathrm{E}-12$ & $6.69 \mathrm{E}-12$ & $4.88 \mathrm{E}-10$ & $4.65 E-12$ & $\begin{array}{c}9.93 \mathrm{E}- \\
08\end{array}$ & $\begin{array}{l}1.00 \mathrm{E} \\
-10 \\
\end{array}$ & 143 & $1 \mathrm{~s}$ \\
\hline
\end{tabular}



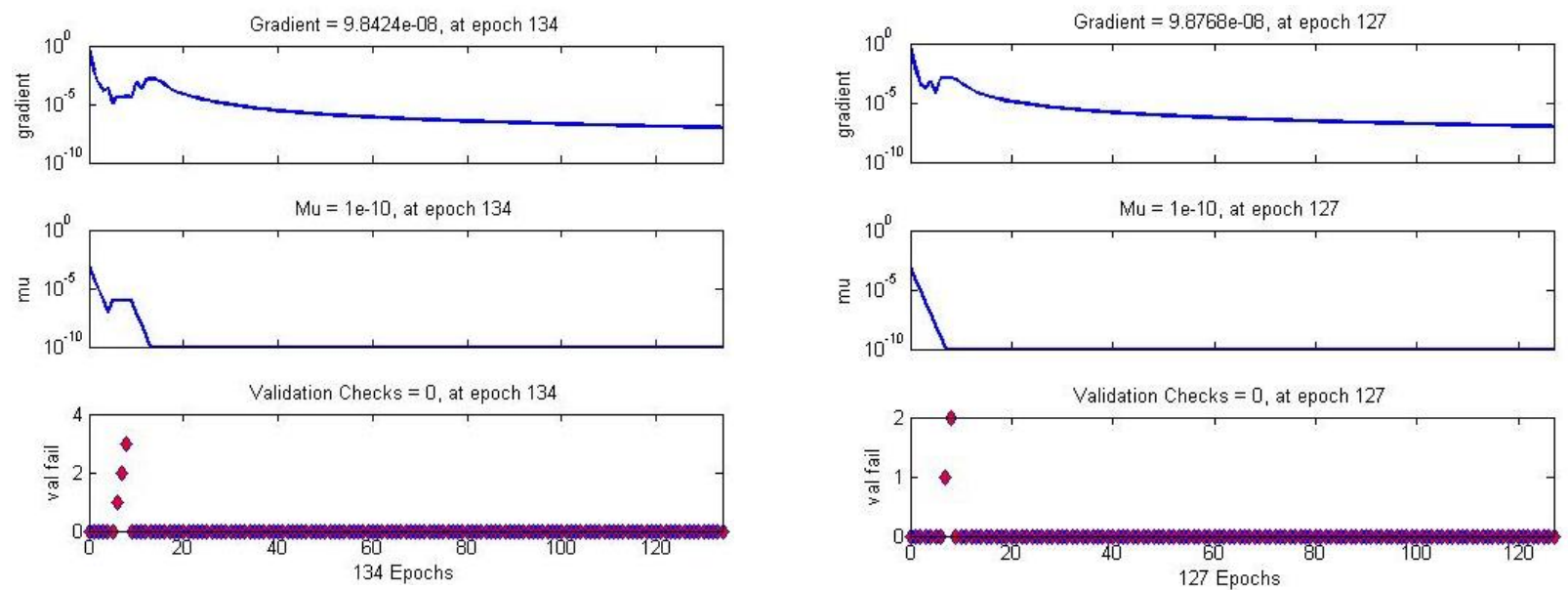

a) Training State of Case 2 of Scenario 1

b) Training State of Case 2 of Scenario 2
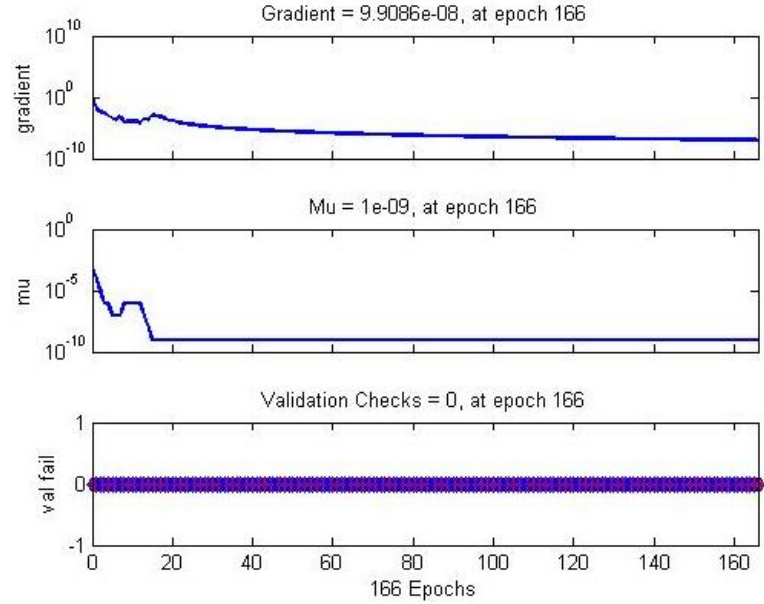

c) Training State of Case 2 of Scenario 3

Figure 5: Transition State of designed BNN-LMA of Case 2 of all the three scenarios of MHD-SNFM

Table 3: Outcomes of BNN-LMA for Scenario 2 of MHD-SNFM

\begin{tabular}{|r|r|l|l|l|l|c|c|c|c|}
\hline \multicolumn{1}{|c|}{ Case } & \multirow{2}{*}{$\begin{array}{c}\text { Hidden } \\
\text { neurons }\end{array}$} & \multicolumn{3}{|c|}{ MSE } & Performance & Grad & Mu & Epochs & Time \\
\cline { 2 - 8 } & & Training & Validation & Testing & & & & & \\
\hline 1 & 10 & $5.58 \mathrm{E}-12$ & $1.39 \mathrm{E}-10$ & $7.72 \mathrm{E}-12$ & $5.58 \mathrm{E}-12$ & $\begin{array}{c}9.87 \mathrm{E}- \\
08\end{array}$ & $\begin{array}{c}1.00 \mathrm{E}- \\
10\end{array}$ & 131 & $1 \mathrm{~s}$ \\
\hline 2 & 10 & $5.58 \mathrm{E}-12$ & $1.79 \mathrm{E}-11$ & $3.08 \mathrm{E}-11$ & $5.58 \mathrm{E}-12$ & $\begin{array}{c}9.88 \mathrm{E}- \\
08\end{array}$ & $\begin{array}{c}1.00 \mathrm{E}- \\
10\end{array}$ & 127 & $2 \mathrm{~s}$ \\
\hline 3 & 10 & $2.72 \mathrm{E}-12$ & $1.47 \mathrm{E}-11$ & $9.83 \mathrm{E}-11$ & $2.72 \mathrm{E}-12$ & $\begin{array}{c}9.90 \mathrm{E}- \\
09\end{array}$ & $\begin{array}{c}1.00 \mathrm{E}- \\
10\end{array}$ & 217 & $2 \mathrm{~s}$ \\
\hline 4 & 10 & $4.29 \mathrm{E}-11$ & $1.85 \mathrm{E}-10$ & $1.00 \mathrm{E}-10$ & $4.29 \mathrm{E}-11$ & $\begin{array}{c}9.98 \mathrm{E}- \\
08\end{array}$ & $\begin{array}{c}1.00 \mathrm{E}- \\
09\end{array}$ & 182 & $1 \mathrm{~s}$ \\
\hline
\end{tabular}



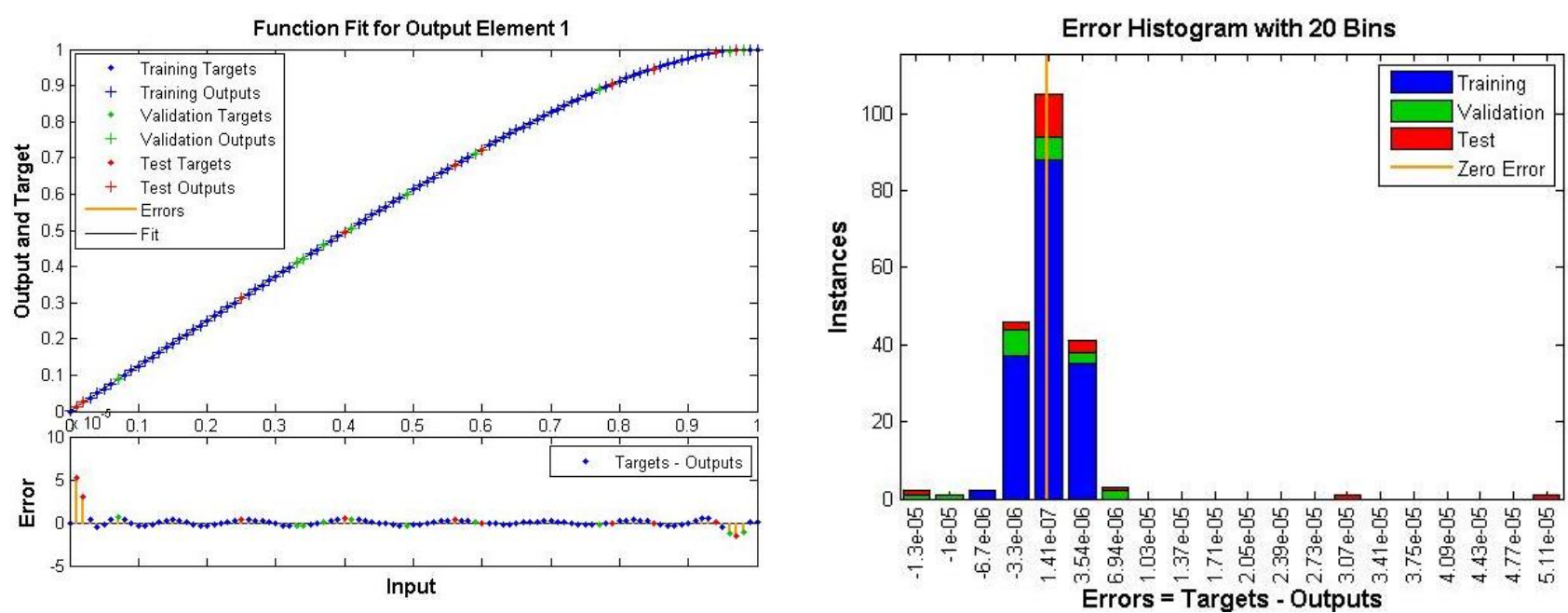

a) Fitness and error histogram of Case 2 of Scenario 1 of BNN-LMA

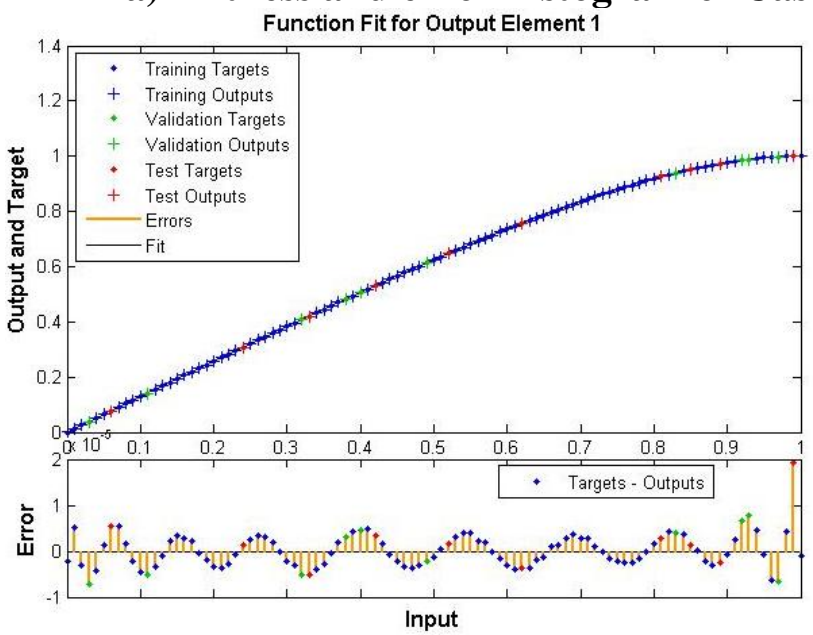

Error Histogram with 20 Bins

b) Fitness and error histogram of Case 2 of Scenario 2 of BNN-LMA

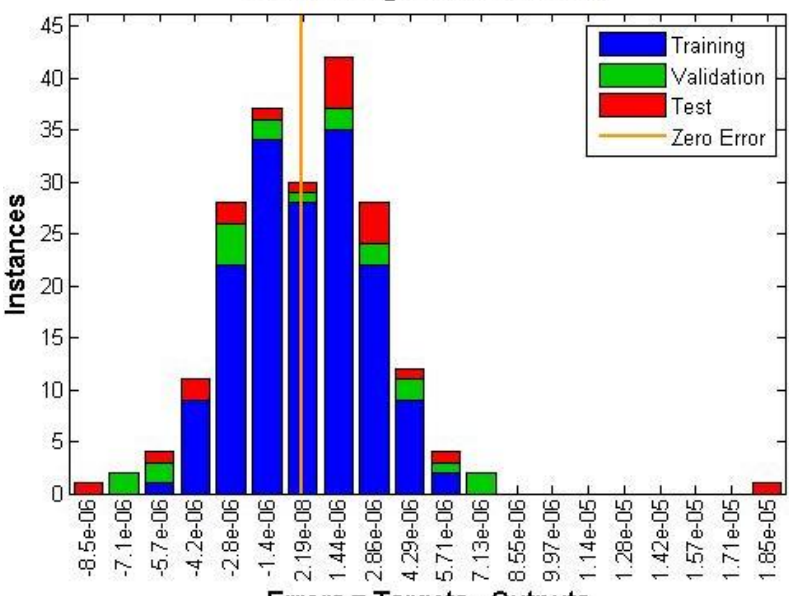

Errors $=$ Targets - Outputs

Function Fit for Output Element 1
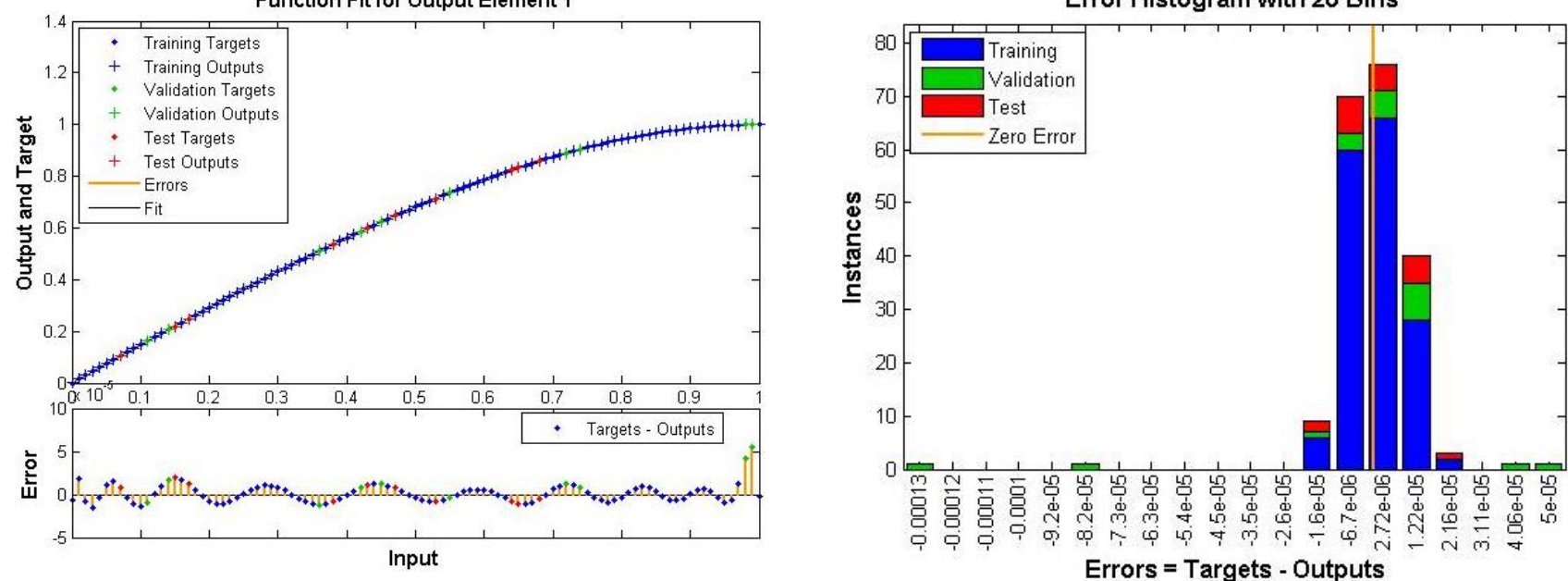

c) Fitness and error histogram of Case 2 of Scenario 3 of BNN-LMA

Figure 6: Function Fitness and error histogram studies of designed BNN-LMA of MHD-SNFM 

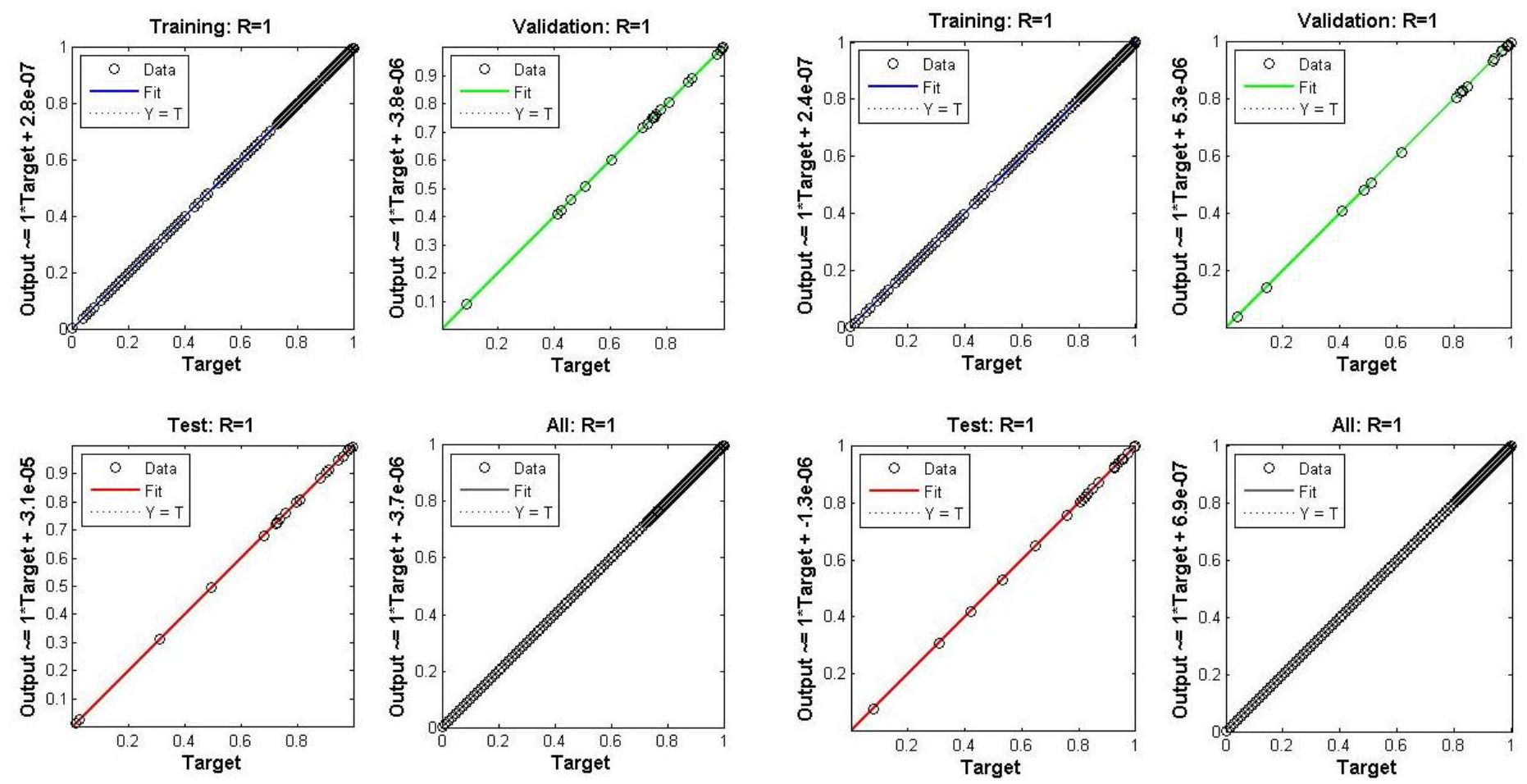

a) Regression of Case 2 of Scenario

b) Regression of Case 2 of Scenario2
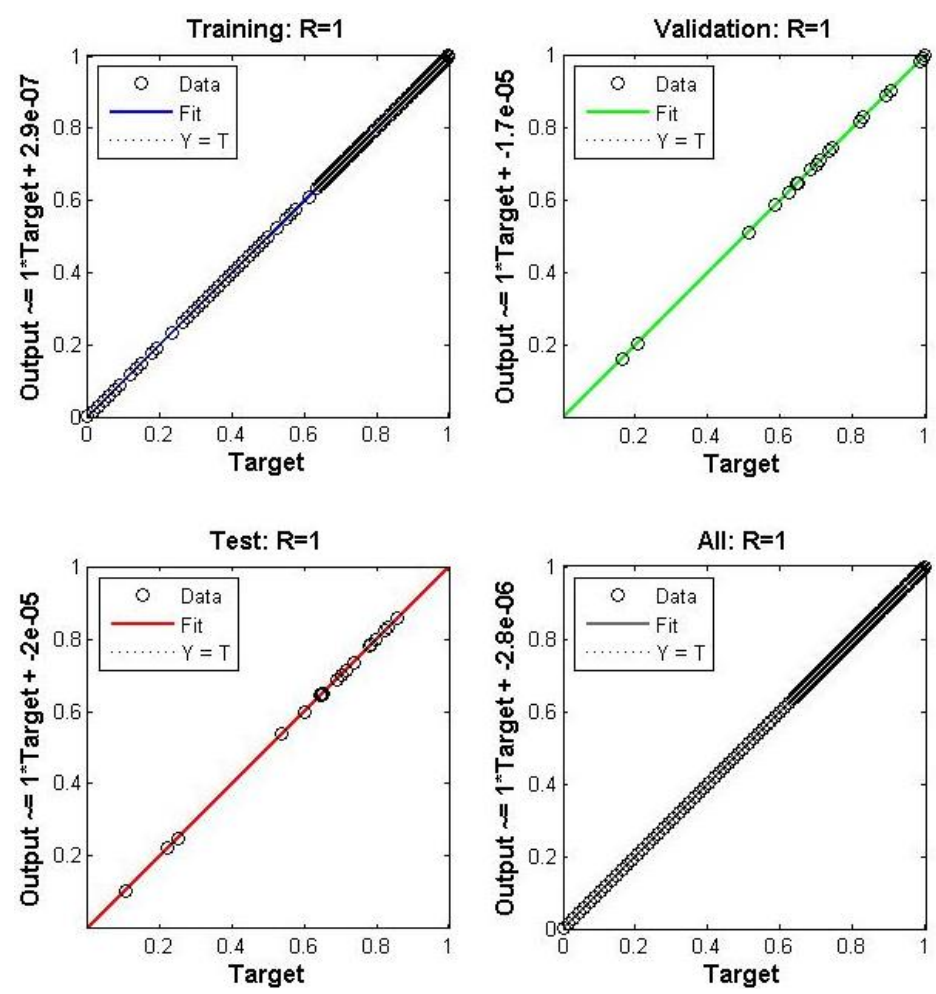

c) Regression of Case 2 of Scenario 3

Figure 7: Regression presentations of BNN-LMA for case 2 of all the Scenarios of MHD-SNFM 
Table 4: Outcomes of BNN-LMA for Scenario 3 of MHD-SNFM

\begin{tabular}{|r|r|c|c|c|c|c|c|c|c|}
\hline \multicolumn{1}{|c|}{ Case } & \multirow{2}{*}{$\begin{array}{c}\text { Hidden } \\
\text { neurons }\end{array}$} & \multicolumn{3}{|c|}{ MSE } & Performance & Grad & Mu & Epochs & Time \\
\cline { 3 - 9 } & & Training & Validation & Testing & & & & & \\
\hline 1 & 10 & $3.95 \mathrm{E}-12$ & $6.28 \mathrm{E}-12$ & $5.44 \mathrm{E}-10$ & $3.95 \mathrm{E}-12$ & $\begin{array}{c}9.89 \mathrm{E}- \\
08\end{array}$ & $\begin{array}{c}1.00 \mathrm{E}- \\
10\end{array}$ & 153 & $1 \mathrm{~s}$ \\
\hline 2 & 10 & $5.49 \mathrm{E}-11$ & $1.52 \mathrm{E}-09$ & $8.98 \mathrm{E}-11$ & $5.49 \mathrm{E}-11$ & $\begin{array}{c}9.91 \mathrm{E}- \\
08\end{array}$ & $\begin{array}{c}1.00 \mathrm{E}- \\
09\end{array}$ & 166 & $1 \mathrm{~s}$ \\
\hline 3 & 10 & $4.92 \mathrm{E}-12$ & $7.40 \mathrm{E}-12$ & $5.11 \mathrm{E}-12$ & $4.92 \mathrm{E}-12$ & $\begin{array}{c}9.93 \mathrm{E}- \\
09\end{array}$ & $\begin{array}{c}1.00 \mathrm{E}- \\
10\end{array}$ & 127 & $1 \mathrm{~s}$ \\
\hline 4 & 10 & $4.67 \mathrm{E}-11$ & $7.30 \mathrm{E}-11$ & $4.81 \mathrm{E}-11$ & $4.67 \mathrm{E}-11$ & $\begin{array}{c}9.92 \mathrm{E}- \\
08\end{array}$ & $\begin{array}{c}1.00 \mathrm{E}- \\
09\end{array}$ & 158 & $1 \mathrm{~s}$ \\
\hline
\end{tabular}

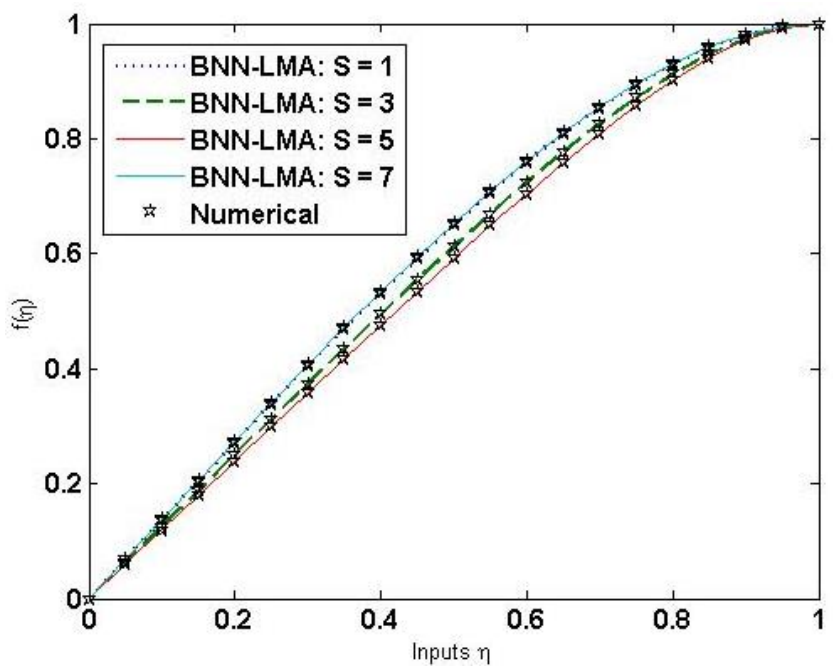

a) Variation of $S$ for $f$

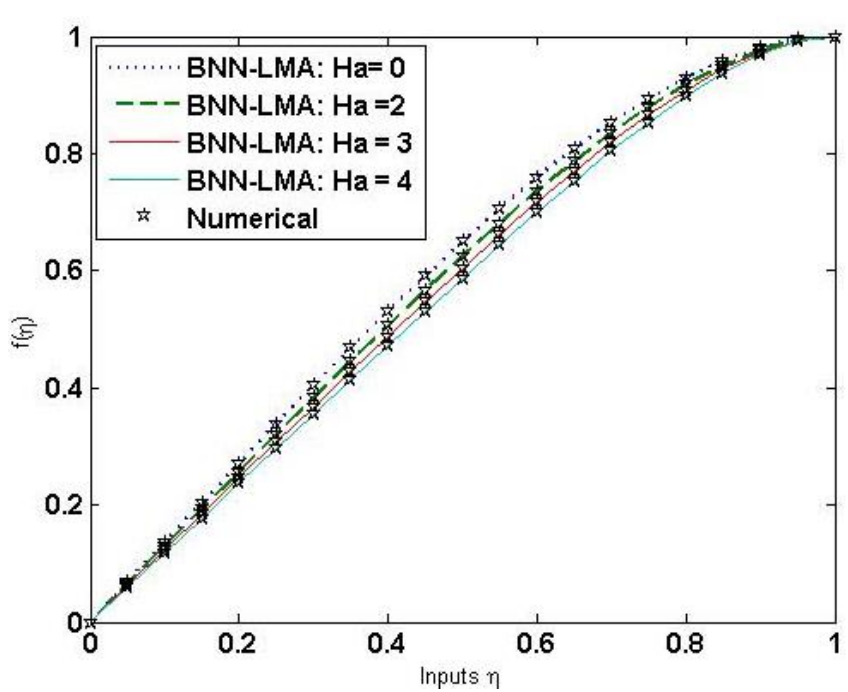

c) Variation of $\mathrm{Ha}$ for $\boldsymbol{f}$

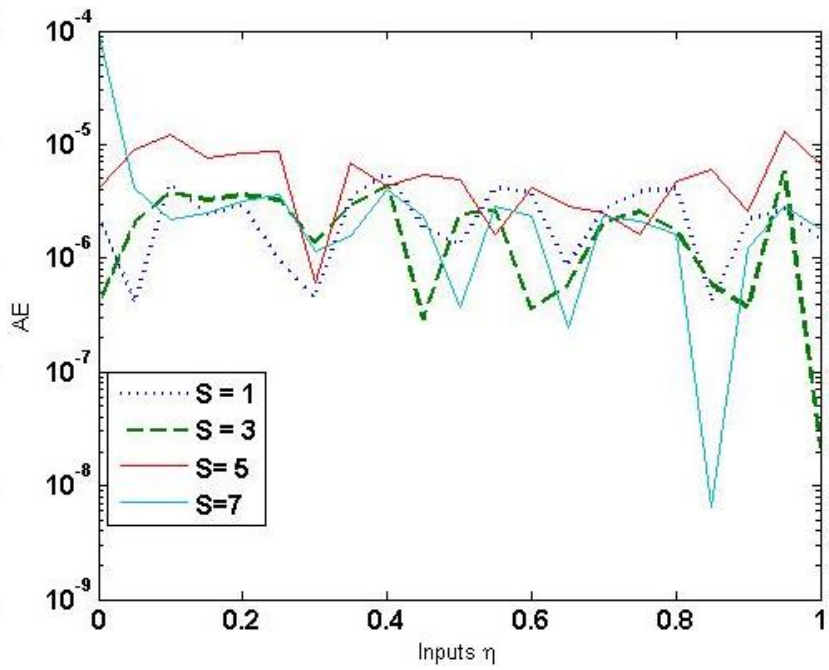

b) Analysis of $\mathrm{AE}$ in variation of $\boldsymbol{S}$ for $\boldsymbol{f}$

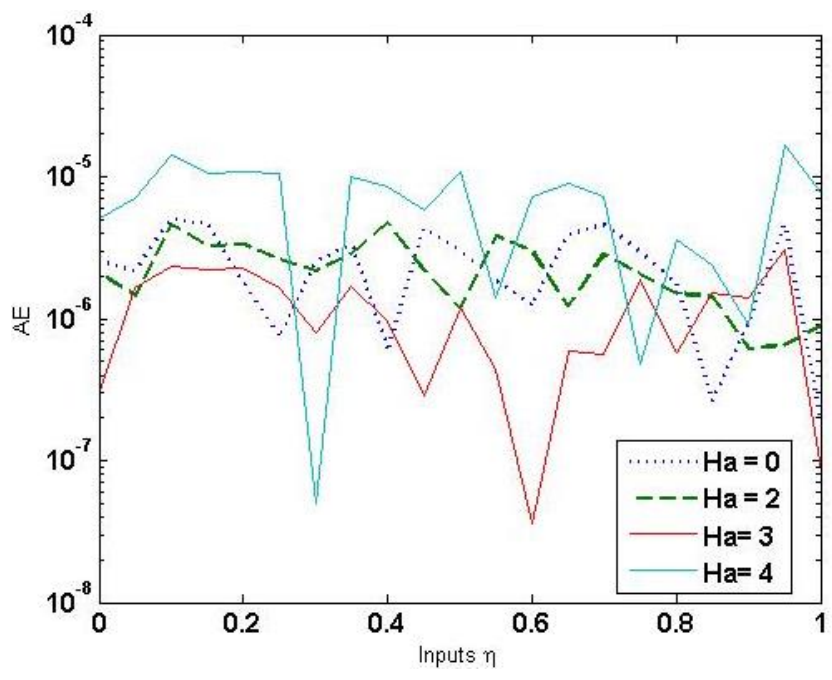

c) Analysis of $\mathrm{AE}$ in variation of $\mathrm{Ha}$ for $f$ 


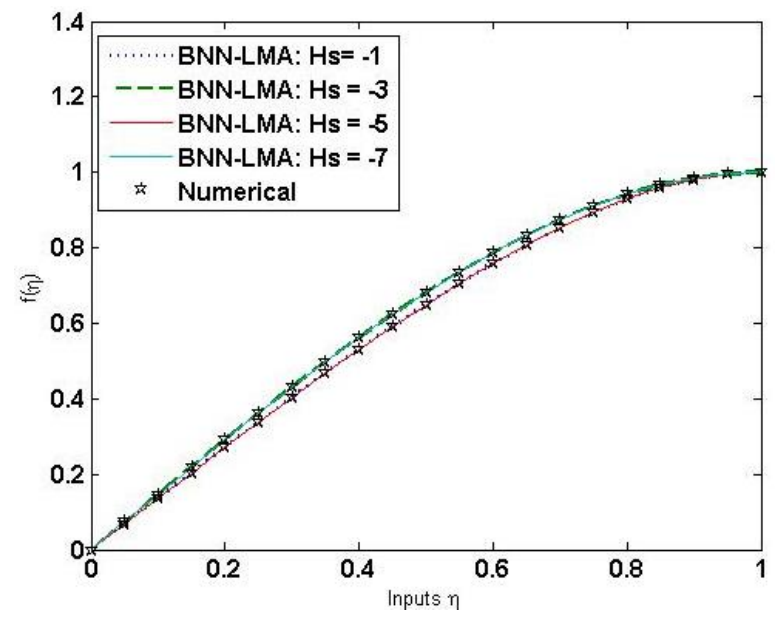

e) Variation of $\boldsymbol{H} s$ for $\boldsymbol{f}$

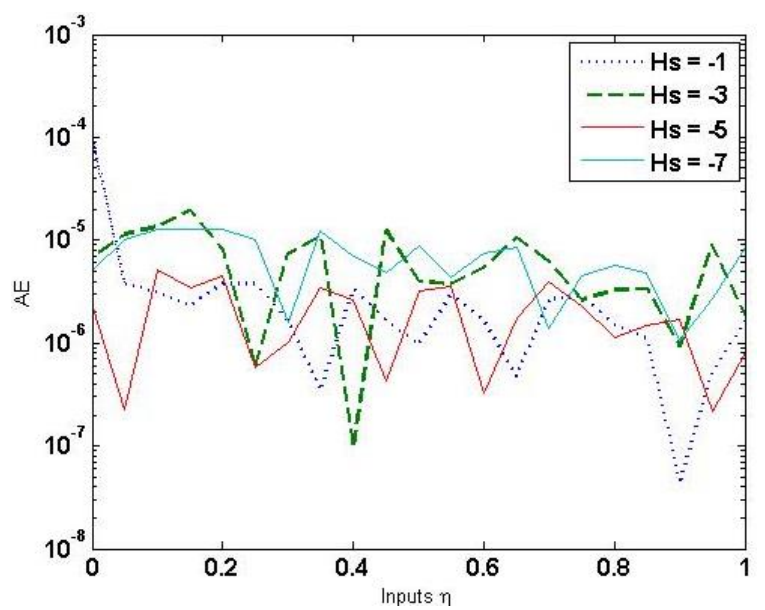

f) Analysis of $\mathrm{AE}$ in variation of $\boldsymbol{H} \boldsymbol{s}$ for $\boldsymbol{f}$

Figure 8: Assessment of BNN-LMA with reference dataset for $f$ of MHD-SNFM

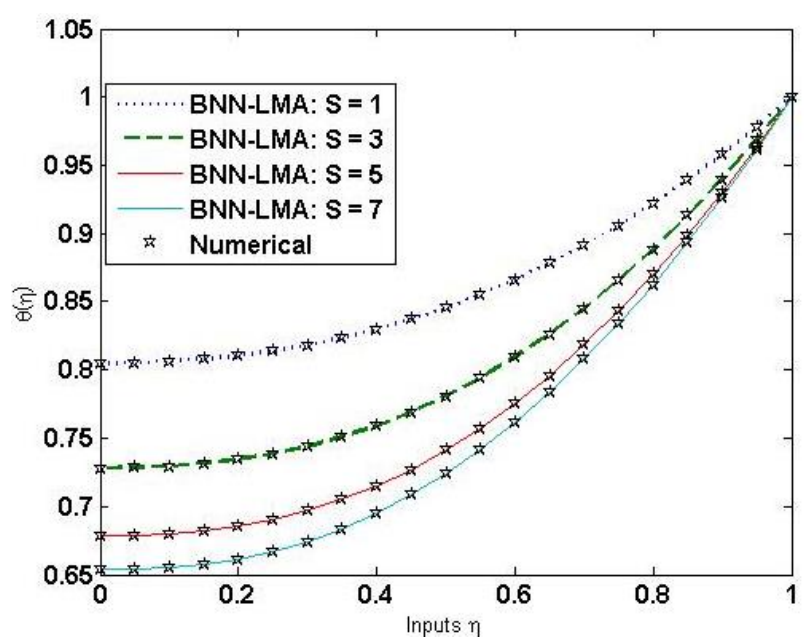

a) Variation of $S$ for $\theta$

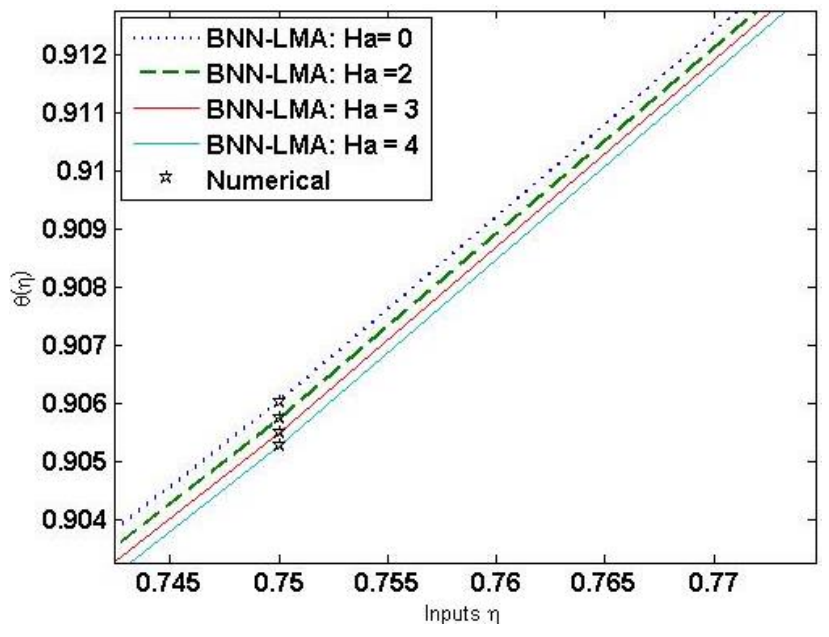

c) Variation of $\mathrm{Ha}$ for $\boldsymbol{\theta}$

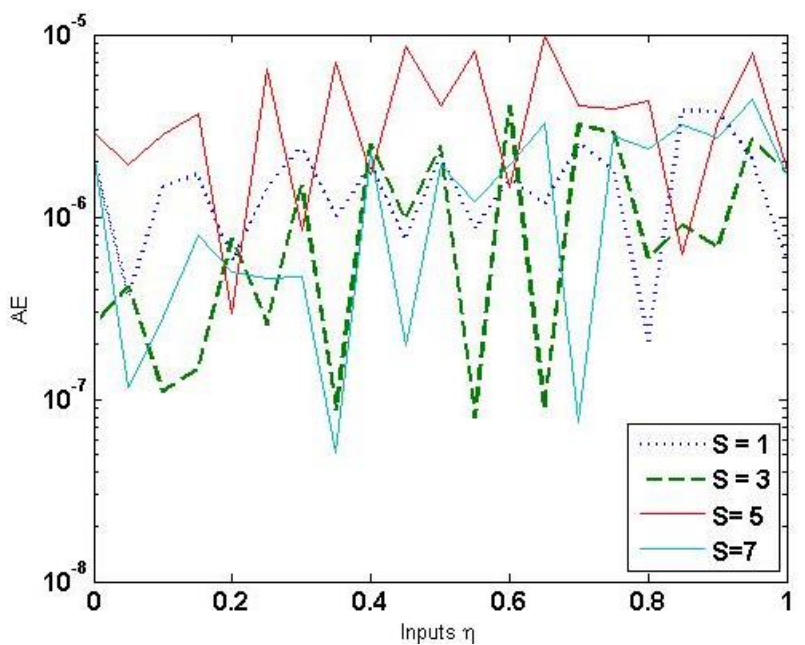

b) Analysis of $\mathrm{AE}$ in variation of $\boldsymbol{S}$ for $\theta$

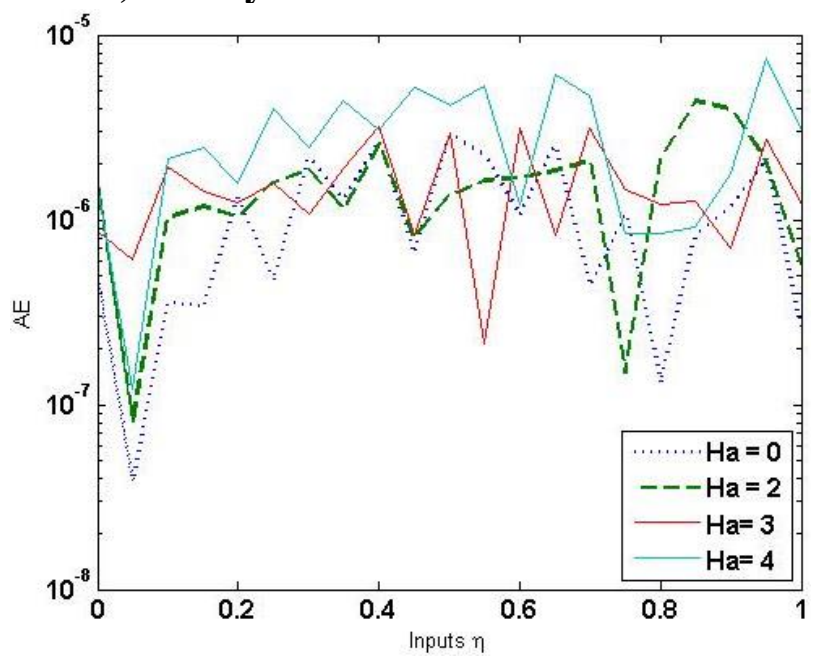

d) Analysis of $\mathrm{AE}$ in variation of $\mathrm{Ha}$ for $\boldsymbol{\theta}$ 


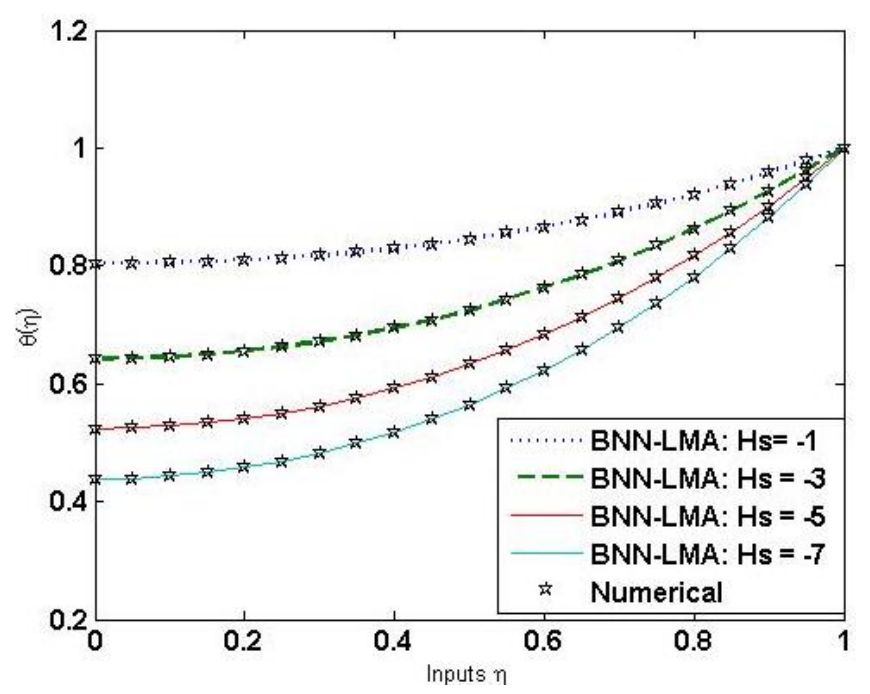

e) Variation of $\boldsymbol{H} \boldsymbol{s}$ for $\boldsymbol{\theta}$

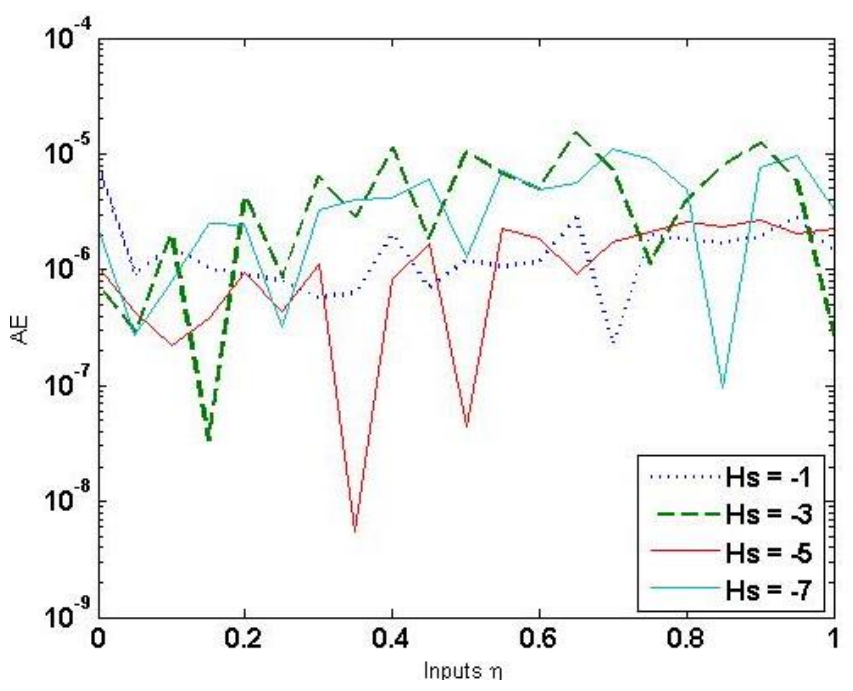

f) Analysis of AE in variation of $H s$ for $\theta$

Figure 9: Assessment of BNN-LMA with reference dataset for $\theta$ of MHD-SNFM

\section{Conclusion:}

The artificial intelligence based backpropagated neural networks with Levenberg-Marquardt algorithm (BNN-LMA) are utilized to interpret the solution of mathematical model of squeezing 2D magneto-hydrodynamic (MHD) nanofluid flow between two parallel plates for different scenarios by variation of squeezing number, Hartmann Number and heat source parameter. The PDEs of MHD squeezing nanofluid flow model are transformed into equivalent ODEs by using similarity variables. Adam numerical solver is utilized to generate the reference dataset of MHDSNFM by variation of different variants. The $80 \%, 10 \%$ and $10 \%$ of the reference data are utilized for training, testing and validation for BNN-LMA. The proposed and reference outcomes verify the correctness of the technique and is further justified through numerical and graphical illustrations of convergence plots of MSE, regression and correlation analysis, and histogram studies.

\section{References:}

1. Choi, S.U. and Eastman, J.A., 1995. Enhancing thermal conductivity of fluids with nanoparticles (No. ANL/MSD/CP-84938; CONF-951135-29). Argonne National Lab., IL (United States).

2. Hayat, T., Sajjad, R., Alsaedi, A., Muhammad, T. and Ellahi, R., 2017. On squeezed flow of couple stress nanofluid between two parallel plates. Results in physics, 7, pp.553-561.

3. Mahmood, M., Asghar, S. and Hossain, M.A., 2007. Squeezed flow and heat transfer over a porous surface for viscous fluid. Heat and mass Transfer, 44(2), pp.165-173. 
4. Siddiqui, A.M., Irum, S. and Ansari, A.R., 2008. Unsteady squeezing flow of a viscous MHD fluid between parallel plates, a solution using the homotopy perturbation method. Mathematical Modelling and Analysis, 13(4), pp.565-576.

5. Shoaib, M., Raja, M.A.Z., Sabir, M.T., Islam, S., Shah, Z., Kumam, P. and Alrabaiah, H., 2020. Numerical investigation for rotating flow of MHD hybrid nanofluid with thermal radiation over a stretching sheet. Scientific Reports, 10(1), pp.1-15.

6. Imran, A., Akhtar, R., Zhiyu, Z., Shoaib, M. and Raja, M.A.Z., 2020. Heat transfer analysis of biological nanofluid flow through ductus efferentes. AIP Advances, 10(3), p.035029.

7. Azimi, M. and Riazi, R., 2018. Magnetohydrodynamic go-water nanofluid flow and heat transfer between two parallel moving disks. Thermal Science, 22(1 Part B), pp.383-390.

8. Azimi, A. and Azimi, M., 2018. Analytical investigation of unsteady CuO nanofluid flow, heat and mass transfer between two parallel disks. Indian Journal of Chemical Technology (IJCT), 25(3), pp.281-286.

9. Umar, M., Sabir, Z., Imran, A., Wahab, A.H., Shoaib, M. and Raja, M.A.Z., 2020. The 3-D flow of Casson nanofluid over a stretched sheet with chemical reactions, velocity slip, thermal radiation and Brownian motion. Thermal Science, 24(5 Part A), pp.2929-2939.

10. Babazadeh, H., Muhammad, T., Shakeriaski, F., Ramzan, M. and Hajizadeh, M.R., 2020. Nanomaterial between two plates which are squeezed with impose magnetic force. J. Therm. Anal. Calorim.

11. Noor, N.A.M., Shafie, S. and Admon, M.A., 2020. Unsteady MHD squeezing flow of Jeffrey fluid in a porous medium with thermal radiation, heat generation/absorption and chemical reaction. Physica Scripta, 95(10), p.105213.

12. Noor, N.A.M., Shafie, S. and Admon, M.A., 2020. Effects of viscous dissipation and chemical reaction on MHD squeezing flow of Casson nanofluid between parallel plates in a porous medium with slip boundary condition. The European Physical Journal Plus, 135(10), pp.1-24.

13. Jasim, A.M., 2020. Analytical approximation of the first grade MHD squeezing fluid flow with slip boundary condition using a new iterative method. Heat Transfer.

14. Bhaskar, K. and Sharma, K., 2020. Unsteady MHD squeezing viscous Casson fluid flow in upright channel with cross-diffusion and thermal radiactive effects. Indian Journal of Physics, pp.1-15.

15. Hosseinzadeh, K., Alizadeh, M. and Ganji, D.D., 2020. Retraction Note: Hydrothermal analysis on MHD squeezing nanofluid flow in parallel plates by analytical method. International Journal of Mechanical and Materials Engineering, 15(1), pp.1-1.

16. Nisar, K.S., Khan, U., Zaib, A., Khan, I. and Baleanu, D., 2020. Numerical Simulation of Mixed Convection Squeezing Flow of a Hybrid Nanofluid Containing Magnetized Ferroparticles in 50\%: 50\% of Ethylene Glycol-Water Mixture Base Fluids Between Two Disks With the Presence of a Non-linear Thermal Radiation Heat Flux. Frontiers in Chemistry, 8.

17. Khan, U., Ahmed, N., Zaidi, Z.A., Asadullah, M. and Mohyud-Din, S.T., 2014. MHD squeezing flow between two infinite plates. Ain Shams Engineering Journal, 5(1), pp.187-192.

18. Hayat, T., Yousaf, A., Mustafa, M. and Obaidat, S., 2012. MHD squeezing flow of second-grade fluid between two parallel disks. International journal for numerical methods in fluids, 69(2), pp.399-410.

19. Ullah, I., Rahim, M.T., Khan, H. and Qayyum, M., 2019. Analysis of various semi-numerical schemes for magnetohydrodynamic (MHD) squeezing fluid flow in porous medium. Propulsion and Power Research, 8(1), pp.69-78.

20. Mustafa, M., Hayat, T. and Obaidat, S., 2012. On heat and mass transfer in the unsteady squeezing flow between parallel plates. Meccanica, 47(7), pp.1581-1589.

21. Shamshuddin, M.D. and Narayana, P.S., 2020. Combined effect of viscous dissipation and Joule heating on MHD flow past a Riga plate with Cattaneo-Christov heat flux. Indian Journal of Physics, 94(9), pp.1385-1394. 
22. AZIMI, A. and AZIMI, M., 2014. Analytical investigation on 2-D unsteady MHD viscoelastic flow between moving parallel plates using RVIM and HPM. Walailak Journal of Science and Technology (WJST), 11(11), pp.955-963.

23. Branch, M. and Mahshahr, I.R.I., 2014. Investigation on the film flow of a third grade fluid on an inclined plane using HPM. Mechanics and Mechanical Engineering, 18(1), pp.5-10.

24. Awan, S.E., Raja, M.A.Z., Gul, F., Khan, Z.A., Mehmood, A. and Shoaib, M., 2020. Numerical Computing Paradigm for Investigation of Micropolar Nanofluid Flow Between Parallel Plates System with Impact of Electrical MHD and Hall Current. Arabian Journal for Science and Engineering, pp.118.

25. Azimi, M. and Riazi, R., 2017. Flow and heat transfer of MHD graphene oxide-water nanofluid between two non-parallel walls. Thermal Science, 21(5), pp.2095-2104.

26. Jadoon, I., Raja, M.A.Z., Junaid, M., Ahmed, A., ur Rehman, A. and Shoaib, M., 2020. Design of evolutionary optimized finite difference based numerical computing for dust density model of nonlinear Van-der Pol Mathieu's oscillatory systems. Mathematics and Computers in Simulation, 181, pp.444-470.

27. Ouyang, C., Akhtar, R., Raja, M.A.Z., Touseef Sabir, M., Awais, M. and Shoaib, M., 2020. Numerical treatment with Lobatto IIIA technique for radiative flow of MHD hybrid nanofluid (Al2O3- $\mathrm{Cu} / \mathrm{H} 2 \mathrm{O}$ ) over a convectively heated stretchable rotating disk with velocity slip effects. AIP Advances, 10(5), p.055122.

28. Ellahi, R., Sait, S.M., Shehzad, N. and Ayaz, Z., 2019. A hybrid investigation on numerical and analytical solutions of electro-magnetohydrodynamics flow of nanofluid through porous media with entropy generation. International Journal of Numerical Methods for Heat \& Fluid Flow.

29. Bhatti, M.M., Shahid, A., Abbas, T., Alamri, S.Z. and Ellahi, R., 2020. Study of activation energy on the movement of gyrotactic microorganism in a magnetized nanofluids past a porous plate. Processes, 8(3), p.328.

30. Khan, L.A., Raza, M., Mir, N.A. and Ellahi, R., 2020. Effects of different shapes of nanoparticles on peristaltic flow of MHD nanofluids filled in an asymmetric channel. Journal of Thermal Analysis and Calorimetry, 140(3), pp.879-890.

31. Shahid, A., Huang, H., Bhatti, M.M., Zhang, L. and Ellahi, R., 2020. Numerical investigation on the swimming of gyrotactic microorganisms in nanofluids through porous medium over a stretched surface. Mathematics, 8(3), p.380.

32. Riaz, A., Zeeshan, A., Bhatti, M.M. and Ellahi, R., 2020. Peristaltic propulsion of Jeffrey nano-liquid and heat transfer through a symmetrical duct with moving walls in a porous medium. Physica A: Statistical Mechanics and its Applications, 545, p.123788.

33. Sarafraz, M.M., Pourmehran, O., Yang, B., Arjomandi, M. and Ellahi, R., 2020. Pool boiling heat transfer characteristics of iron oxide nano-suspension under constant magnetic field. International Journal of Thermal Sciences, 147, p.106131.

34. Zeeshan, A., Hussain, F., Ellahi, R. and Vafai, K., 2019. A study of gravitational and magnetic effects on coupled stress bi-phase liquid suspended with crystal and Hafnium particles down in steep channel. Journal of Molecular Liquids, 286, p.110898.

35. Sabir, Z., Wahab, H.A., Umar, M., Sakar, M.G. and Raja, M.A.Z., 2020. Novel design of Morlet wavelet neural network for solving second order Lane-Emden equation. Mathematics and Computers in Simulation, 172, pp.1-14.

36. Faisal, F., Shoaib, M. and Raja, M.A.Z., 2020. A new heuristic computational solver for nonlinear singular Thomas-Fermi system using evolutionary optimized cubic splines. The European Physical Journal Plus, 135(1), pp.1-29.

37. Sabir, Z., Manzar, M.A., Raja, M.A.Z., Sheraz, M. and Wazwaz, A.M., 2018. Neuro-heuristics for nonlinear singular Thomas-Fermi systems. Applied Soft Computing, 65, pp.152-169. 
38. Raja, M.A.Z., Mehmood, A., Khan, A.A. and Zameer, A., 2019. Integrated intelligent computing for heat transfer and thermal radiation-based two-phase MHD nanofluid flow model. Neural Computing and Applications, pp.1-33.

39. Mehmood, A., Zameer, A., Ling, S.H. and Raja, M.A.Z., 2018. Design of neuro-computing paradigms for nonlinear nanofluidic systems of MHD Jeffery-Hamel flow. Journal of the Taiwan Institute of Chemical Engineers, 91, pp.57-85.

40. Raja, M.A.Z., Manzar, M.A., Shah, S.M. and Chen, Y., 2020. Integrated intelligence of fractional neural networks and sequential quadratic programming for Bagley-Torvik systems arising in fluid mechanics. Journal of Computational and Nonlinear Dynamics, 15(5).

41. Ahmad, I., Ilyas, H., Urooj, A., Aslam, M.S., Shoaib, M. and Raja, M.A.Z., 2019. Novel applications of intelligent computing paradigms for the analysis of nonlinear reactive transport model of the fluid in soft tissues and microvessels. Neural Computing and Applications, 31(12), pp.9041-9059.

42. Raja, M.A.Z., Ahmed, T. and Shah, S.M., 2017. Intelligent computing strategy to analyze the dynamics of convective heat transfer in MHD slip flow over stretching surface involving carbon nanotubes. Journal of the Taiwan Institute of Chemical Engineers, 80, pp.935-953.

43. Raja, M.A.Z., Farooq, U., Chaudhary, N.I. and Wazwaz, A.M., 2016. Stochastic numerical solver for nanofluidic problems containing multi-walled carbon nanotubes. Applied Soft Computing, 38, pp.561586.

44. Sabir, Z., Umar, M., Guirao, J.L., Shoaib, M. and Raja, M.A.Z., 2020. Integrated intelligent computing paradigm for nonlinear multi-singular third-order Emden-Fowler equation. Neural Computing and Applications, pp.1-20.

45. Sabir, Z., Raja, M.A.Z., Shoaib, M. and Aguilar, J.G., 2020. FMNEICS: fractional Meyer neuroevolution-based intelligent computing solver for doubly singular multi-fractional order Lane-Emden system. Computational and Applied Mathematics, 39(4), pp.1-18.

46. Sabir, Z., Raja, M.A.Z., Umar, M. and Shoaib, M., 2020. Design of neuro-swarming-based heuristics to solve the third-order nonlinear multi-singular Emden-Fowler equation. The European Physical Journal Plus, 135(6), pp.1-17.

47. Bukhari, A.H., et al., 2020. Fractional neuro-sequential ARFIMA-LSTM for financial market forecasting. IEEE Access, 8, pp.71326-71338.

48. Bukhari, A.H., Sulaiman, M., Raja, M.A.Z., Islam, S., Shoaib, M. and Kumam, P., 2020. Design of a hybrid NAR-RBFs neural network for nonlinear dusty plasma system. Alexandria Engineering Journal, 59(5), pp.3325-3345.

49. Umar, M., et al., 2020. A stochastic computational intelligent solver for numerical treatment of mosquito dispersal model in a heterogeneous environment. The European Physical Journal Plus, 135(7), pp.1-23.

50. Sabir, Z., Raja, M.A.Z., Guirao, J.L. and Shoaib, M., 2020. Integrated intelligent computing with neuro-swarming solver for multi-singular fourth-order nonlinear Emden-Fowler equation. Computational and Applied Mathematics, 39(4), pp.1-18.

51. Jadoon, I., Ahmed, A., ur Rehman, A., Shoaib, M. and Raja, M.A.Z., 2020. Integrated meta-heuristics finite difference method for the dynamics of nonlinear unipolar electrohydrodynamic pump flow model. Applied Soft Computing, 97, p.106791.

52. Ahmad, I., Raja, M.A.Z., Ramos, H., Bilal, M. and Shoaib, M., 2020. Integrated neuro-evolution-based computing solver for dynamics of nonlinear corneal shape model numerically. Neural Computing and Applications, pp.1-17.

53. Umar, M., et al., 2020. A Stochastic Intelligent Computing with Neuro-Evolution Heuristics for Nonlinear SITR System of Novel COVID-19 Dynamics. Symmetry, 12(10), p.1628.

54. Cheema, T.N., Raja, M.A.Z., Ahmad, I., Naz, S., llyas, H. and Shoaib, M., 2020. Intelligent computing with Levenberg-Marquardt artificial neural networks for nonlinear system of COVID-19 epidemic model for future generation disease control. The European Physical Journal Plus, 135(11), pp.1-35. 
55. Salehi, S., Nori, A., Hosseinzadeh, K. and Ganji, D.D., 2020. Hydrothermal analysis of MHD squeezing mixture fluid suspended by hybrid nanoparticles between two parallel plates. Case Studies in Thermal Engineering, p.100650.

Acknowledgements

"The authors acknowledge the financial support provided by the Center of Excellence in Theoretical and Computational Science (TaCS-CoE), KMUTT". Moreover, this research project is supported by Thailand Science Research and Innovation (TSRI) Basic Research Fund: Fiscal year 2021 under project number 64A306000005.

\section{Author Contributions}

M.S, M.A.Z.R and P.K modeled and solved the problem. M.S and I.F wrote the manuscript. Z.S, M.A.Z.R and P.K contributed in the numerical computations and plotting the graphical results. All authors finalized the manuscript after its internal evaluation.

\section{Additional Information}

Competing Interests: The authors declare no competing interests 


\section{Figures}
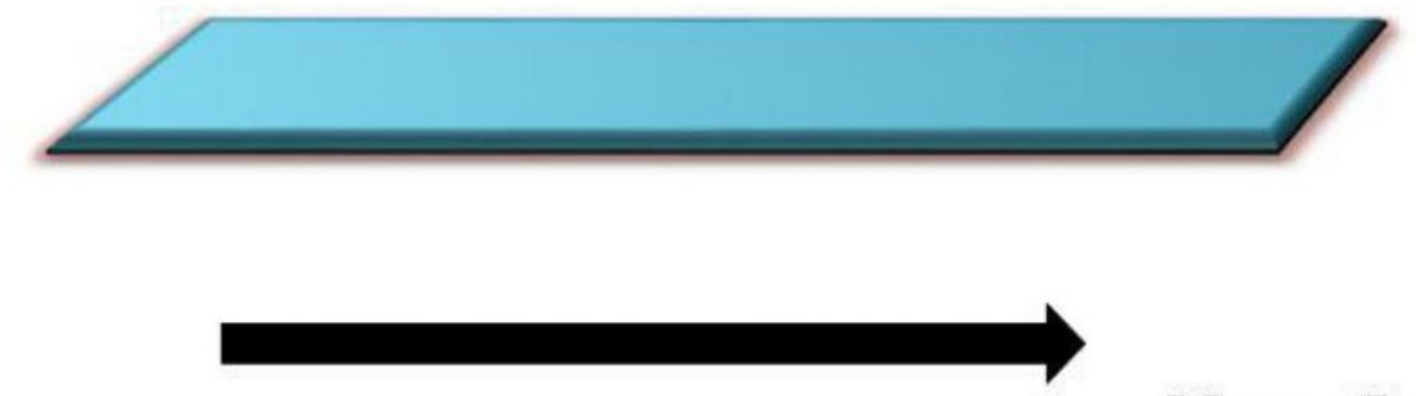

Nanofluid

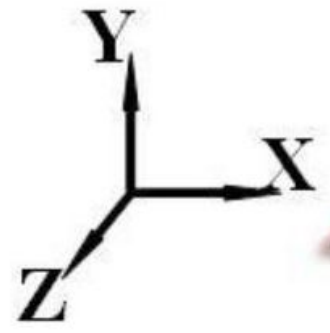

Flow

Figure 1

MHD Squeezing fluid flow between two parallel plates

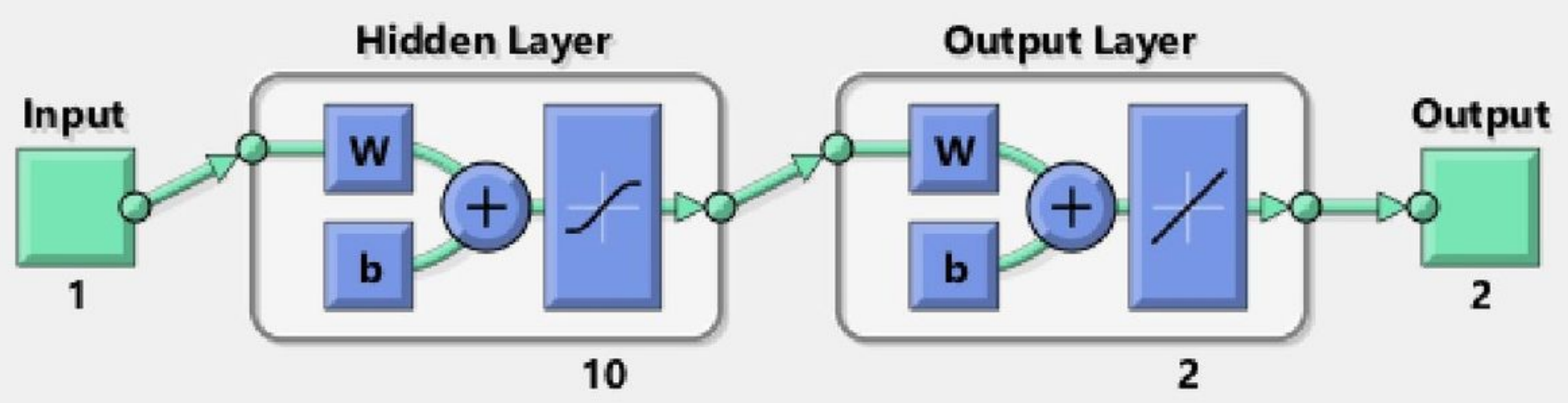

Figure 2

Neural Network for designed BNN-LMA of MHD-SNFM 


\section{The Problem Development}

MHD squeezing fluid suspended by nanoparticles between two parallel plates

System of PDEs representing

MHD-SNFM is converted into system of ODEs

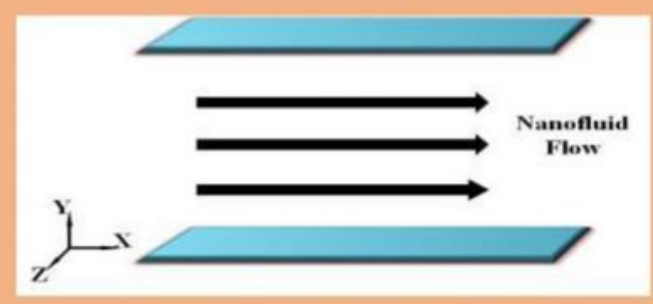

Geometry of the Problem

\section{Step 2:}

\section{Design Scheme for BNN-LMA of proposed MHD-SNFM}

Adam Numerical Outcomes:

Adam numerical solver is utilized to generate the reference dataset by variation of squeezing number.

Hartmann number and heat source parameter of MHD-SNFM
Backpropagated neural networks:

Artificial intelligence based numerical computing technique is devel oped by using neural networks

backpropagated with Levenberg-Marquardt algorithm.

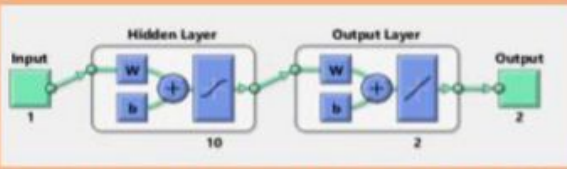

Neural Netw ork for designed BNN-LMA of MHD-SNFM

\section{Analysis of Reference Dataset}

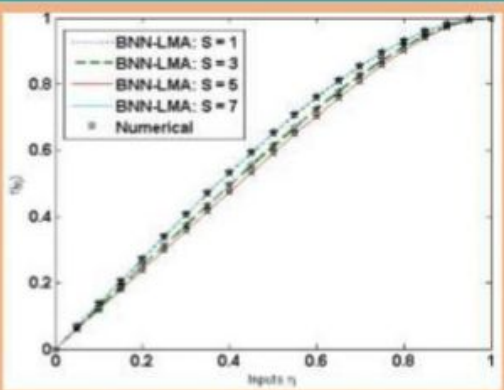

Outcomes with comparison

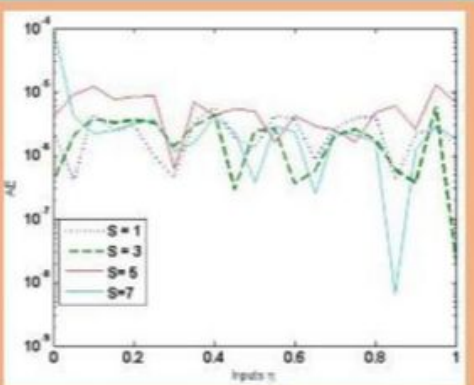

Error Analysis

\section{Figure 3}

Flow Chart of MHD Squeezing fluid flow model 


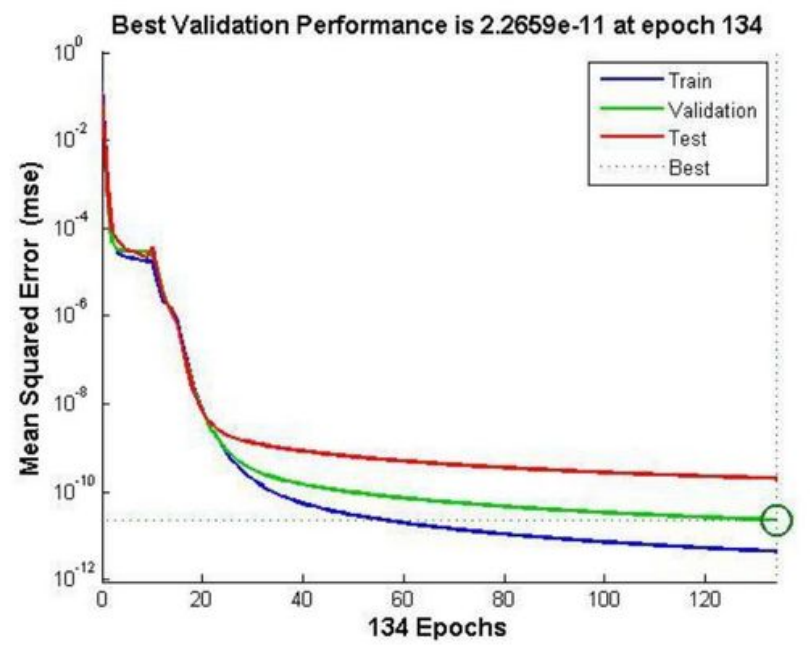

a) Results of MSE for Case 2 of Scenario 1

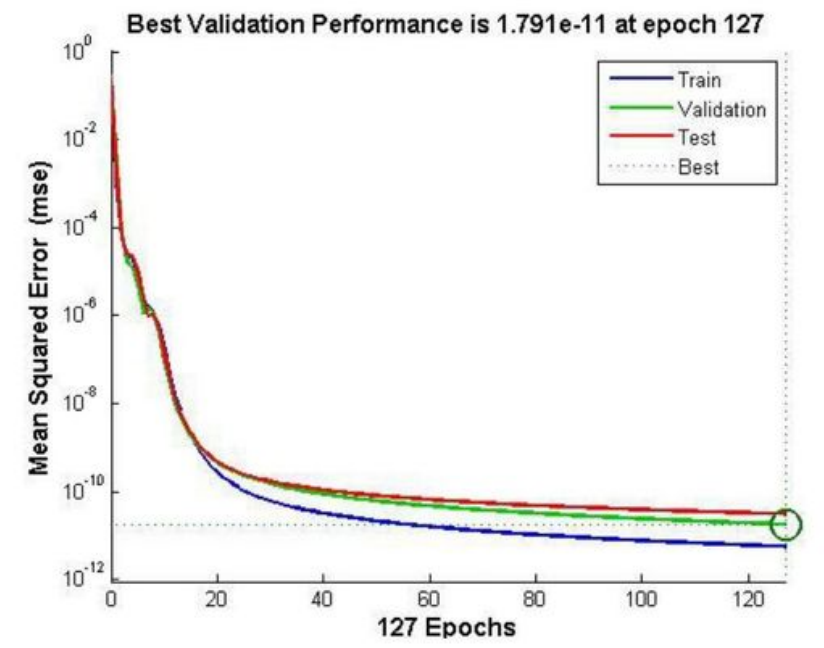

b) Results of MSE for Case 2 of Scenario 2

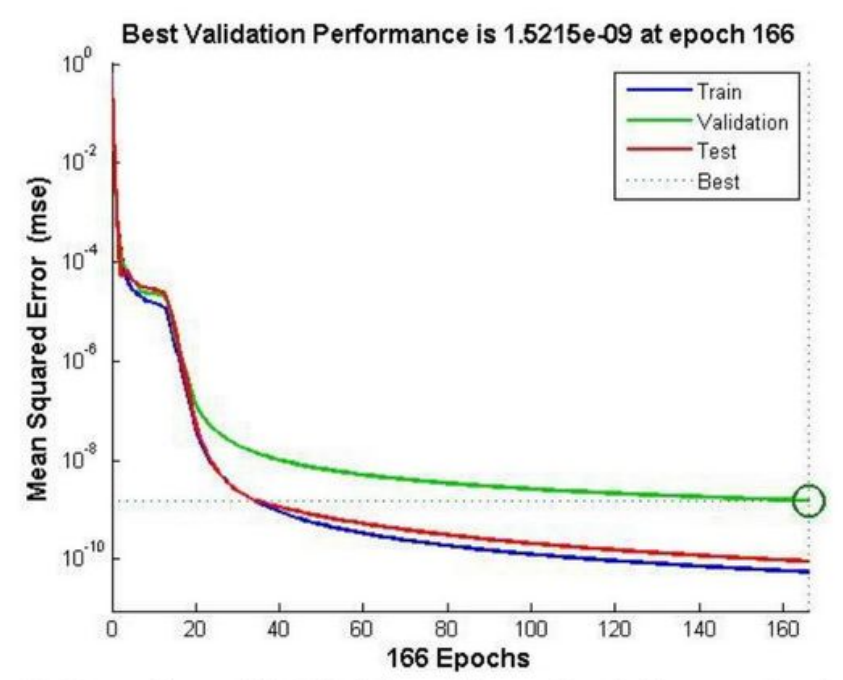

c) Results of MSE for Case 2 of Scenario 3

\section{Figure 4}

Performance on MSE of designed BNN-LMA of case 2 of all the scenarios of MHD-SNFM 

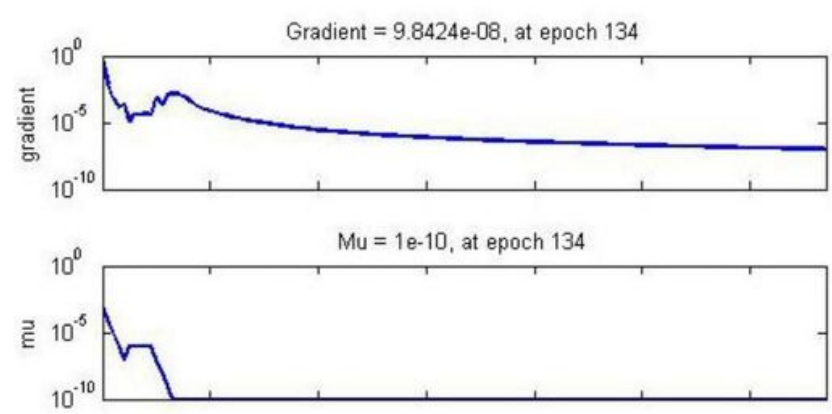

Validation Checks $=0$, at epoch 134

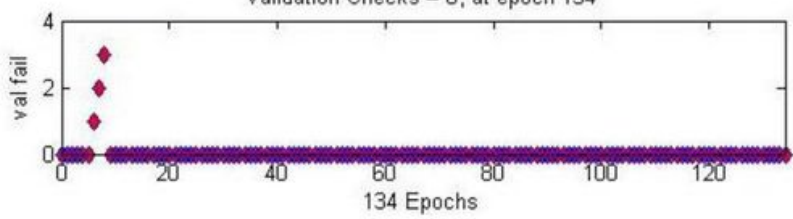

a) Training State of Case 2 of Scenario 1
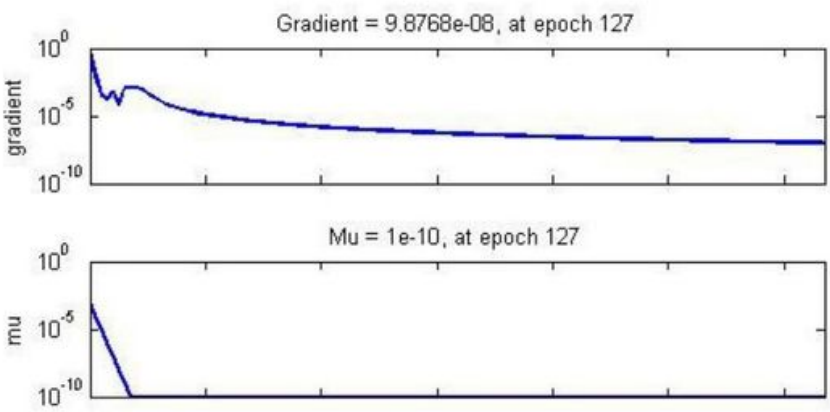

Validation Checks $=0$, at epoch 127

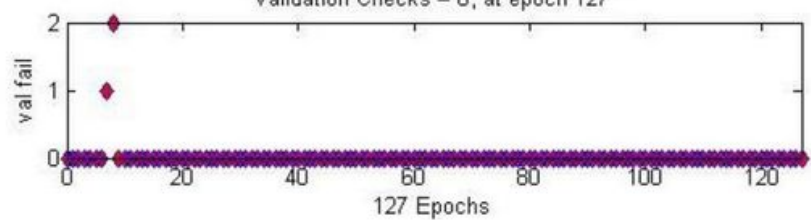

b) Training State of Case 2 of Scenario 2
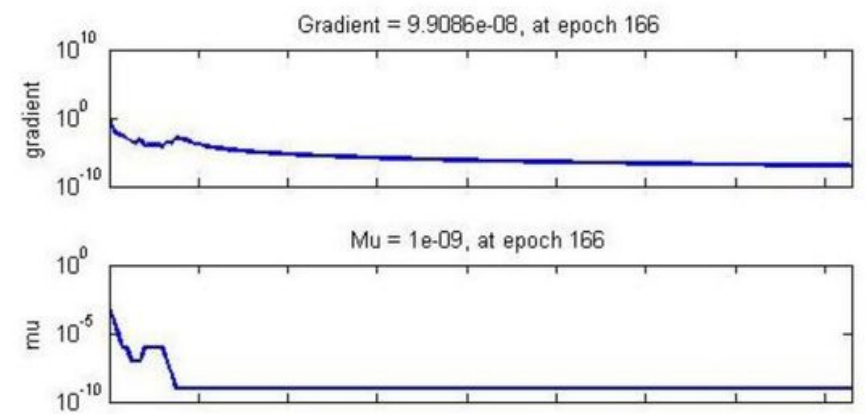

Validation Checks $=0$, at epoch 166

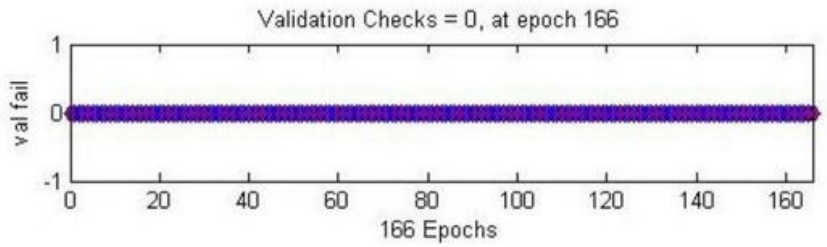

c) Training State of Case 2 of Scenario 3

Figure 5

Transition State of designed BNN-LMA of Case 2 of all the three scenarios of MHD-SNFM 

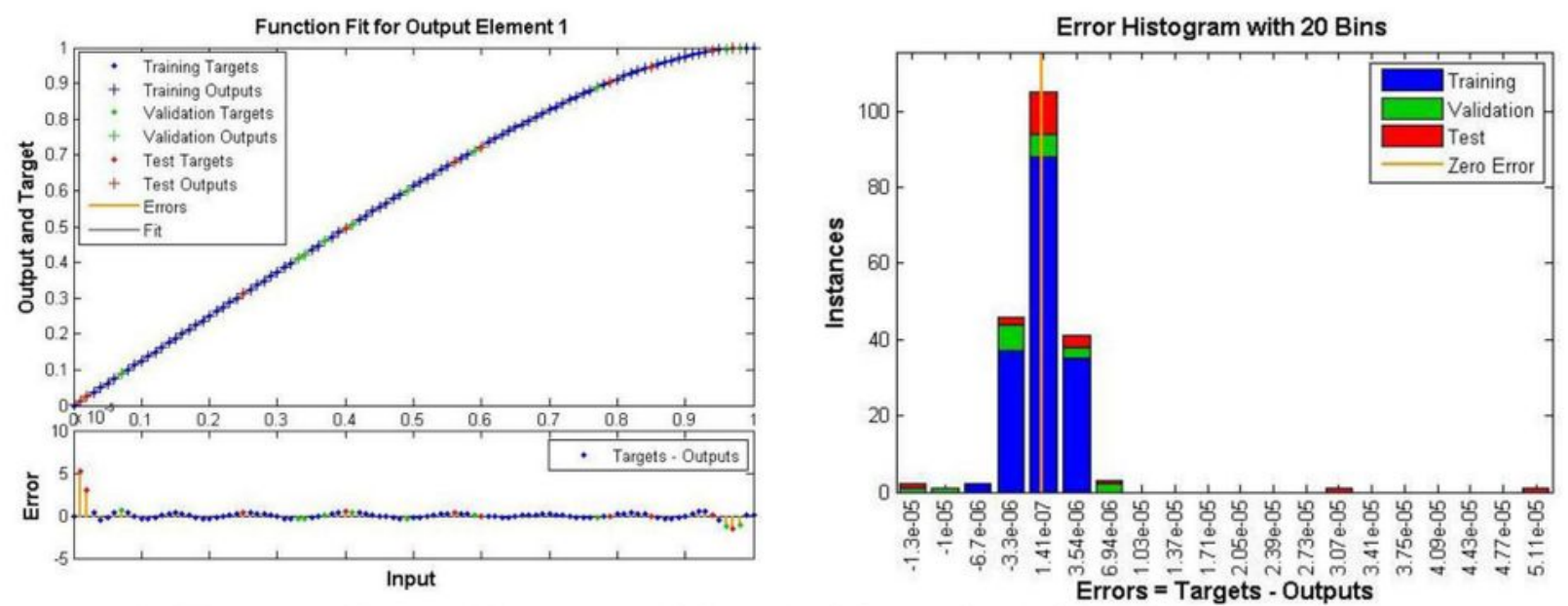

a) Fitness and error histogram of Case 2 of Scenario 1 of BNN-LMA
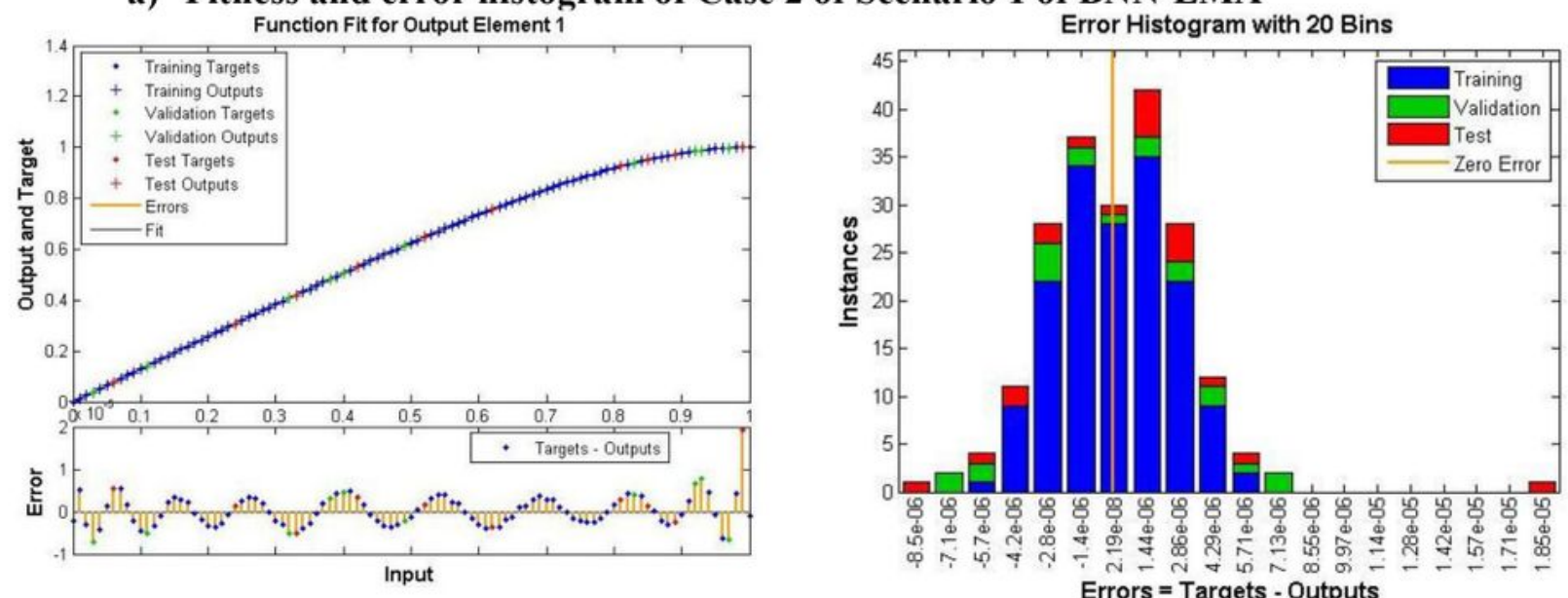

b) Fitness and error histogram of Case 2 of Scenario 2 of BNN-LMA
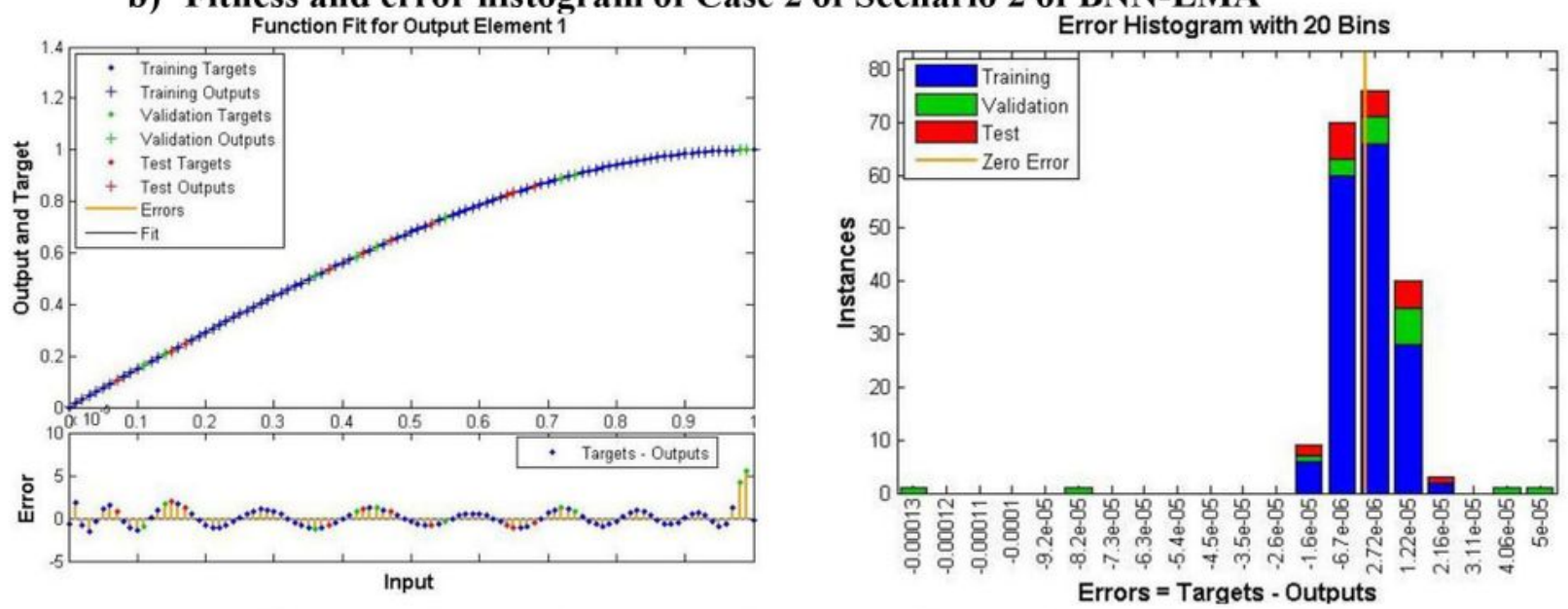

c) Fitness and error histogram of Case 2 of Scenario 3 of BNN-LMA

\section{Figure 6}

Function Fitness and error histogram studies of designed BNN-LMA of MHD-SNFM 

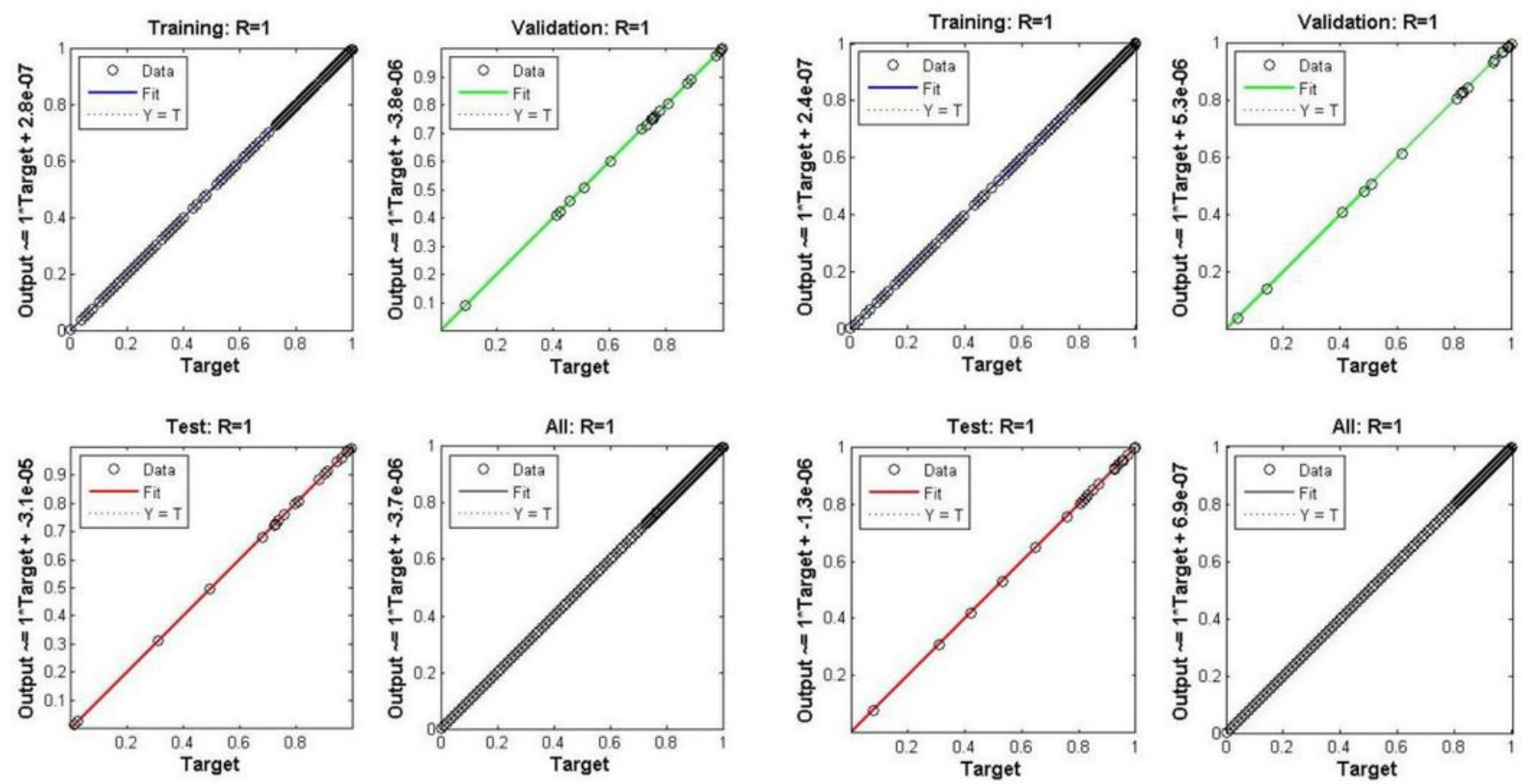

a) Regression of Case 2 of Scenario

b) Regression of Case 2 of Scenario2
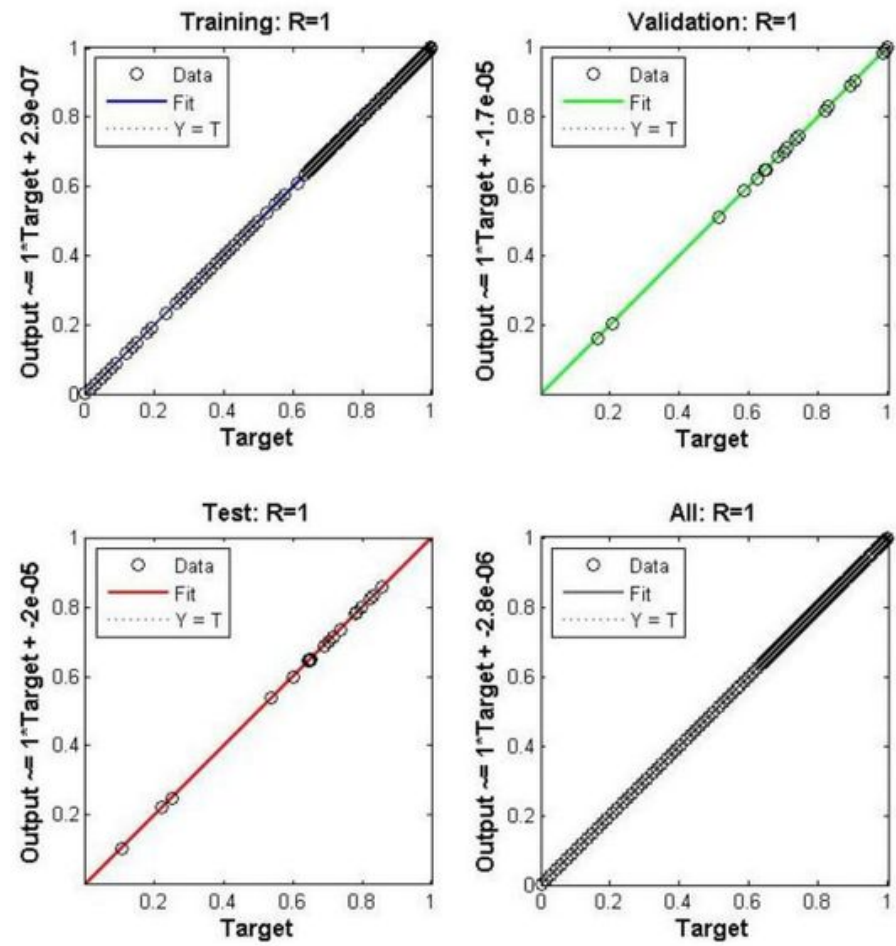

c) Regression of Case 2 of Scenario 3

Figure 7

Regression presentations of BNN-LMA for case 2 of all the Scenarios of MHD-SNFM 


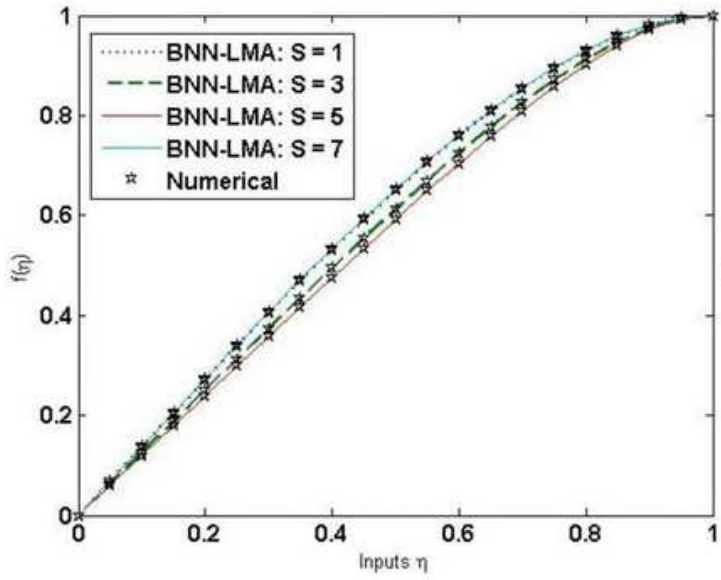

a) Variation of $S$ for $f$

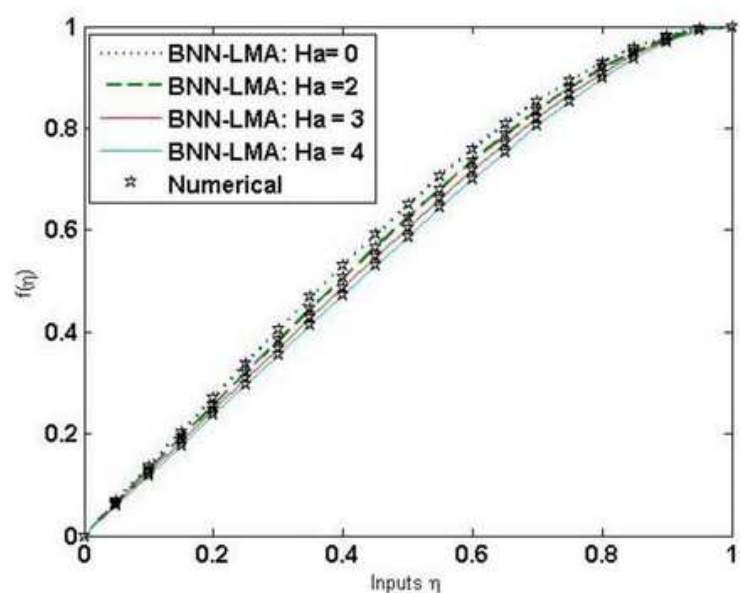

c) Variation of $\mathrm{Ha}$ for $\boldsymbol{f}$

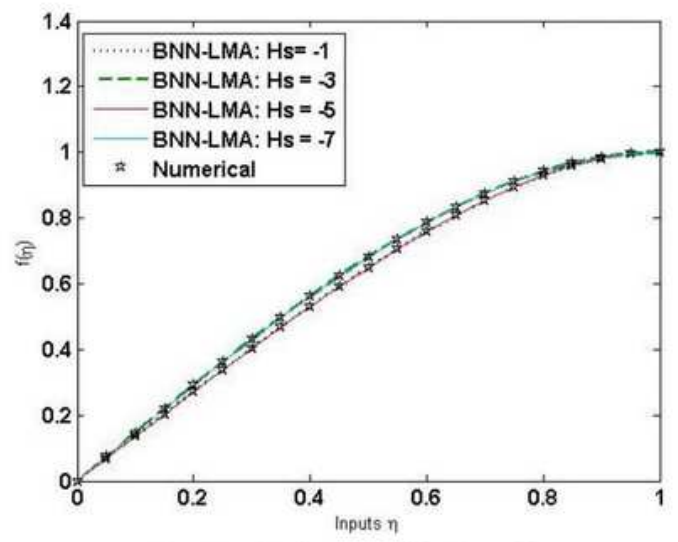

e) Variation of $\boldsymbol{H} s$ for $f$

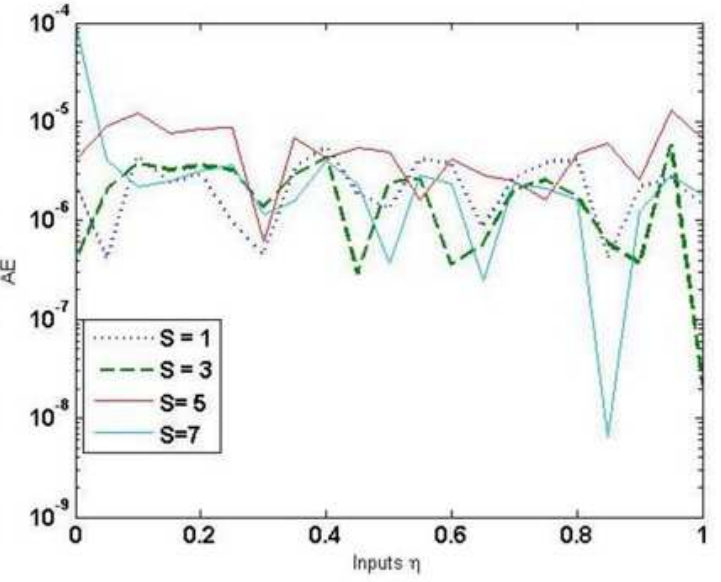

b) Analysis of AE in variation of $S$ for $f$

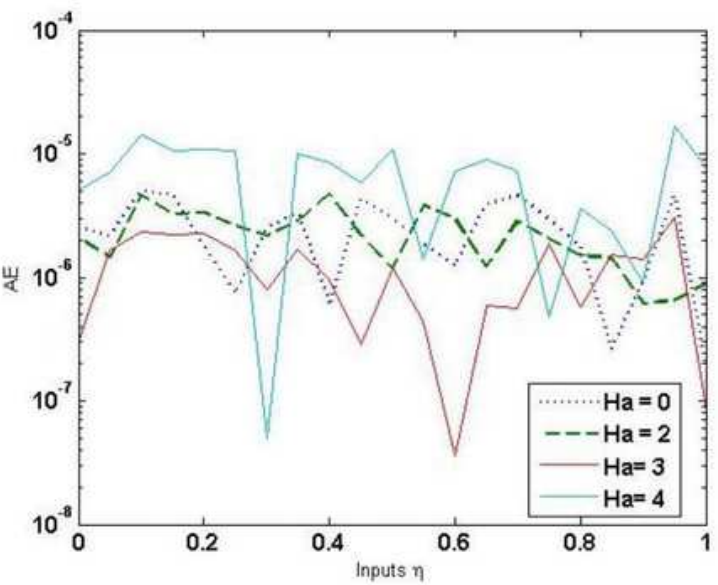

c) Analysis of $\mathrm{AE}$ in variation of $\mathrm{Ha}$ for $\boldsymbol{f}$

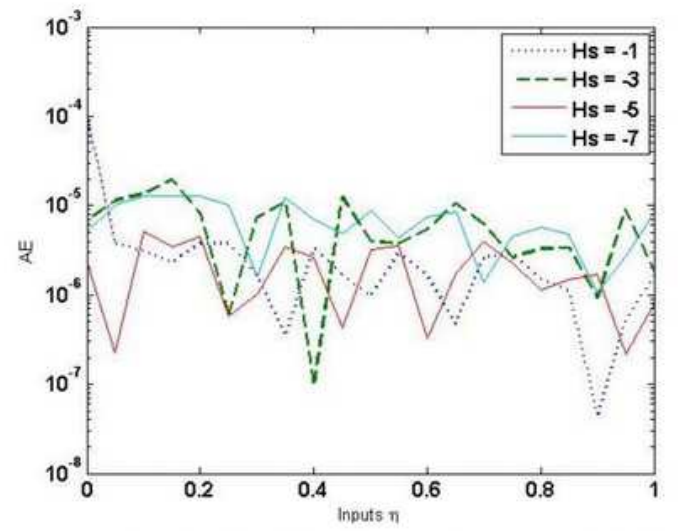

f) Analysis of $\mathrm{AE}$ in variation of $\boldsymbol{H} \boldsymbol{s}$ for $\boldsymbol{f}$

\section{Figure 8}

Assessment of BNN-LMA with reference dataset for $f$ of MHD-SNFM 


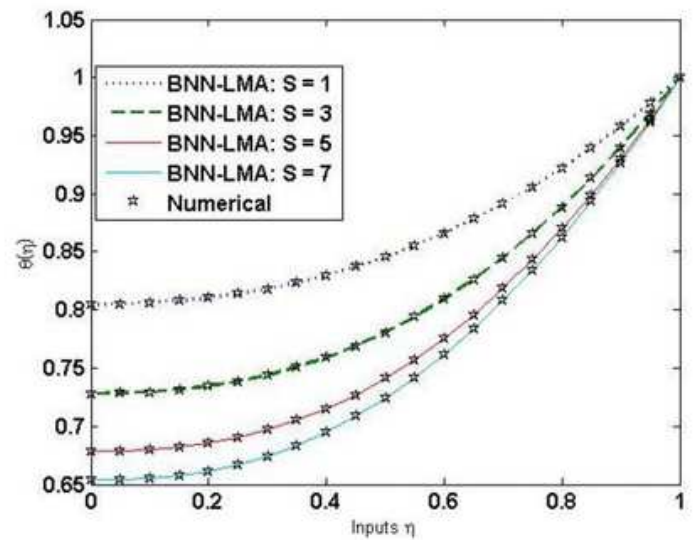

a) Variation of $S$ for $\theta$

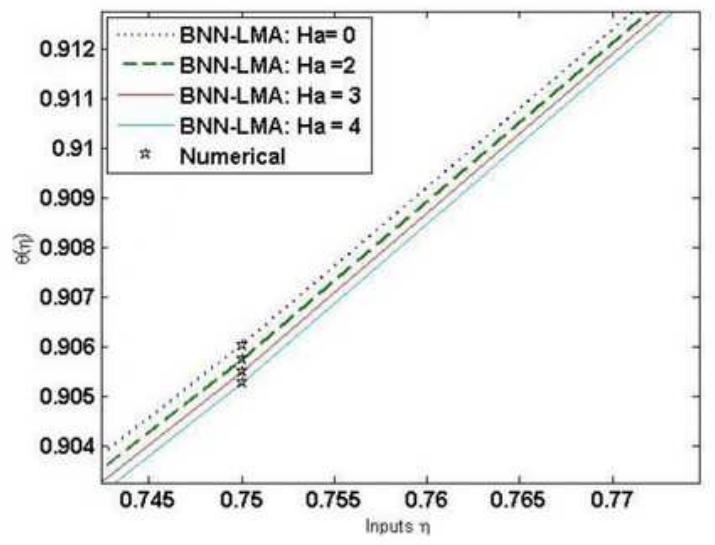

c) Variation of $\mathrm{Ha}$ for $\boldsymbol{\theta}$

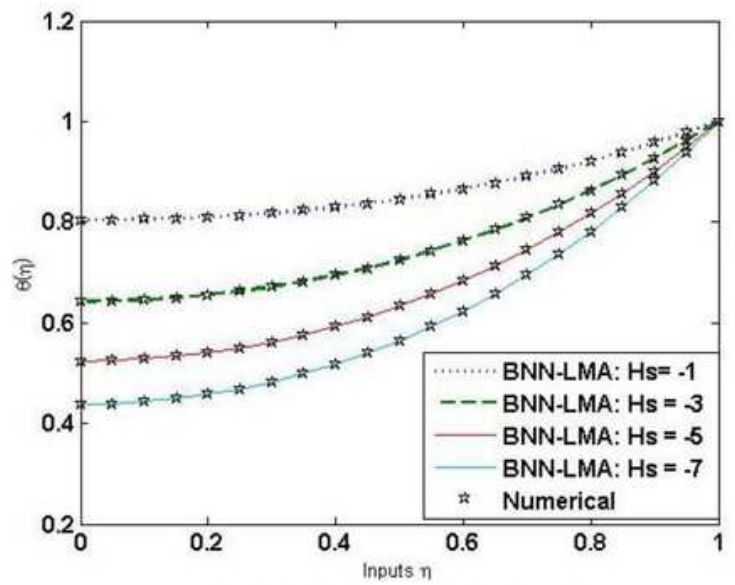

e) Variation of $\boldsymbol{H s}$ for $\boldsymbol{\theta}$

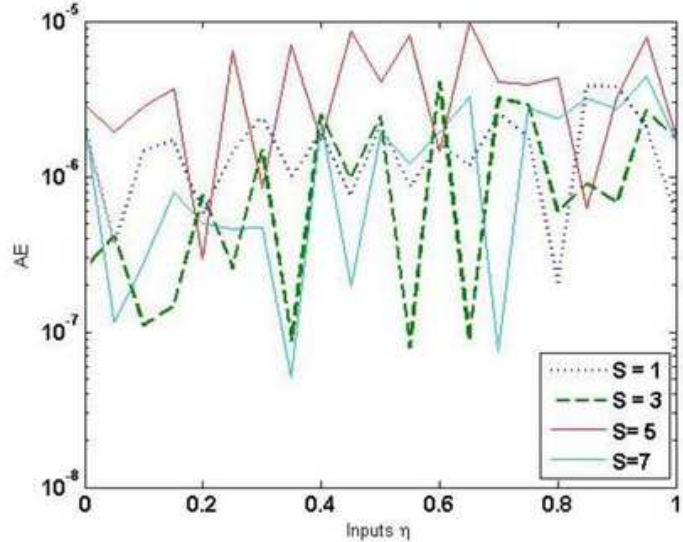

b) Analysis of AE in variation of $S$ for $\theta$

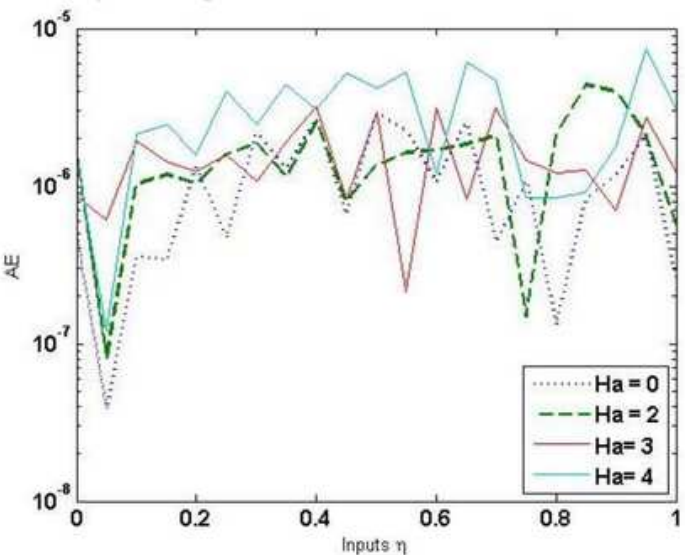

d) Analysis of $\mathrm{AE}$ in variation of $\mathrm{Ha}$ for $\boldsymbol{\theta}$

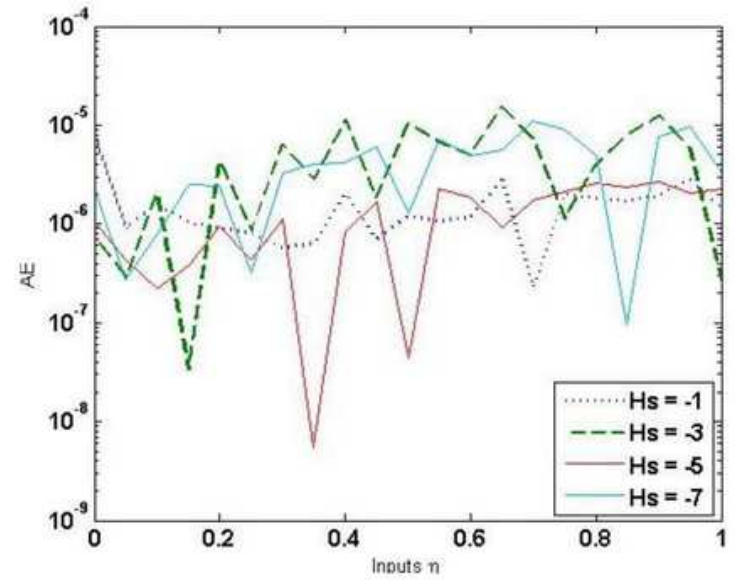

f) Analysis of $\mathrm{AE}$ in variation of $\mathrm{Hs}$ for $\boldsymbol{\theta}$

\section{Figure 9}

Assessment of BNN-LMA with reference dataset for $\theta$ of MHD-SNFM 